\title{
The measurement of Gear Transmission Error as an NVH indicator: Theoretical discussion and industrial application via low-cost digital encoders to an all-electric vehicle gearbox
}

\author{
Antonio Palermo ${ }^{\mathrm{a}, \mathrm{b}, \mathrm{c}}$, Laurent Britte ${ }^{\mathrm{a}, *}$, Karl Janssens $^{\mathrm{a}}$, Domenico Mundo ${ }^{\mathrm{b}}$, Wim Desmet ${ }^{\mathrm{c}, \mathrm{d}}$ \\ a SIEMENS PLM - Simulation and Test Solutions, Digital Factory Division, Interleuvenlaan 68, 3001 Leuven, Belgium \\ ' Universita' della Calabria, Dept. of Mechanical, Energy and Management Engineering, Via P. Bucci, 46C, 87036 Rende, Italy \\ ${ }^{\mathrm{c}} \mathrm{KU}$ Leuven, Department of Mechanical Engineering, Celestijnenlaan 300B, 3001 Leuven, Belgium \\ ${ }^{\mathrm{d}}$ Member of Flanders Make, Belgium
}

\section{A R T I C L E I N F O}

\section{Article history:}

Received 16 October 2017

Received in revised form 11 February 2018

Accepted 5 March 2018

Available online 30 March 2018

\section{Keywords:}

Gear Transmission Error

Elapsed Time Method

Electric vehicle

Low cost encoders

Torsional vibration

\begin{abstract}
A B S T R A C T
Gear Transmission Error (TE) is often considered as the main cause of gear whine. TE represents the difference between the perfectly kinematic transmission of motion and the one actually achieved. TE vibrations are extremely small and pose significant measurement challenges. This article demonstrates how low-cost digital encoders can be successfully used together with the Elapsed Time Method to simplify TE measurement with respect to the traditional Direct Method. A precision gear pair test rig is exploited to compare the two methods from a theoretical and an experimental point of view. Following the observations drawn from such comparison, a measuring chain is set up to validate the proposed procedure on a real case all-electric vehicle gearbox. It is shown how TE represents a useful gearbox NVH indicator and how it can be used to support gear microgeometry design.
\end{abstract}

(c) 2018 Elsevier Ltd. All rights reserved.

\section{Introduction}

Gearboxes are used in a wide variety of applications (vehicles, helicopters, aircrafts, wind turbines, industrial machinery) as a solution to transfer power from a generator to a user which has significantly different torque and speed characteristics. Gearboxes are also known to play a critical role for acoustic comfort and durability in such applications. The present article identifies gear Transmission Error (TE) as a metric to describe the noise and vibration characteristic of a gearbox and provides a methodology to accurately measure it. Although the discussion is of general applicability, here the focus is on the specific relevance of TE measurement for cylindrical involute gears and on the suitability and application of the measurement methodology to a real-case all-electric vehicle gearbox. Electrification is in fact one of the most important trends in today's automotive industry and poses a series of unprecedented challenges. One of these is related to the interiors acoustic comfort, which becomes dominated especially by the gearbox given the absence of masking noise from the internal combustion engine. The contributions of the present article will be positioned through this introduction into the frame of gearbox NVH issues, with a few references to simulation in order to establish a link towards physical causes; then the state of the art for TE measurement will be discussed. The article will continue with the analysis of TE as an insightful NVH indicator/metric

\footnotetext{
* Corresponding author.

E-mail address: laurent.britte@siemens.com (L. Britte).
} 


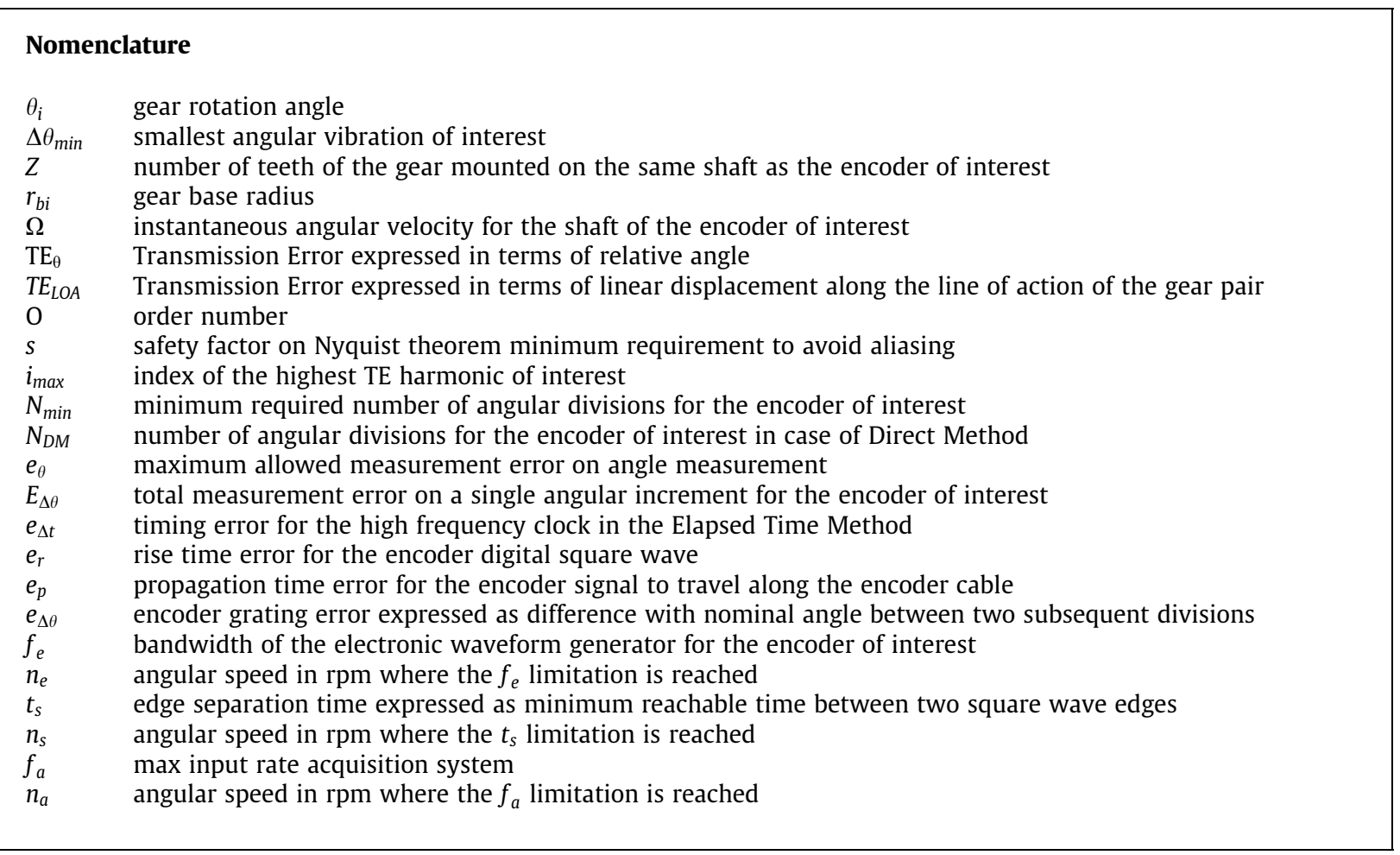

for a gearbox, then with a comparison of the Elapsed Time Method (ETM) and the Direct Method (DM) both from a theoretical and an instrumentation standpoint and finally with the discussion of measurement results on a precision gear pair test rig and the real-case all-electric vehicle power unit.

\subsection{Transmission error and gearbox NVH issues}

Gearbox noise emission can be either structure born or airborne. In fact, torsional vibrations travel across the driveline and become coupled to lateral vibrations at gear meshes, giving rise to undesired dynamic response through bearings and support locations. Such undesired response can be due to either amplification at resonances or strong excitation sources; sources can be classified into two major categories: external and internal.

External excitation sources are due to input or output torque irregularities and usually lead to contact loss, impacts and to the related broadband noise known as gear rattle. Rattle issues are uncommon for electric vehicles, since electric motors deliver a very smooth torque application. The classical example of gear rattle in the automotive field is in fact related to diesel engines running in idle or lightly loaded conditions. In such conditions, the engine torque ripple can overcome the torque transmitted by the gearbox so that the teeth come out of contact [1]. Such problem is usually successfully described and solved thanks to numerical simulations which are able to correctly describe the torque ripple and the torsional dynamic properties of the driveline (1D models). In fact, for rattle problems, it is sufficient to describe rotors by rigid-body inertia moments and gear meshes by constant stiffness coefficients [2,3]. The estimation of damping represents the most difficult technical challenge and it is usually performed by tuning coefficients on experimental data after that the resonance frequencies of interest are correctly captured. From a measurement point of view, driveline torsional vibrations due to load irregularity are relatively high in amplitude and do not require particularly tight measurement accuracy [4]. A variety of instruments and techniques is available for such measurement [5].

Internal excitation sources are mostly due to gear meshing [6]. They generate harmonic oscillations of the transmitted torque and lead typically to tonal noise known as gear whine. Gear whine represents the main concern for acoustic comfort in an electric vehicle, since its tonality can be considerably prominent on the interior noise floor. Whine problems are extremely challenging both from a simulation and from a measurement point of view. For what concerns simulations, the involved physical phenomena are intrinsically three-dimensional: the distribution of contact pressure on tooth surfaces during the meshing process determines how load is transferred when a new tooth pair enters into contact or leaves contact. A smooth load transfer is crucial to reduce gear meshing vibrations and is achieved only through intentional and well-designed tooth surface microgeometry modifications [7,8]. Such microgeometry modifications make the difference between a quiet and a noisy gearbox, therefore they must be carefully optimized. Furthermore, gears are fully coupled -and are interacting 
with- static and dynamic deflections at connection points (e.g. bearings, splines, elastomeric couplings) and at the supporting structure, requiring the solution of a system-level problem with flexible bodies [9]. The solution of such problem is intrinsically stiff on a spatial and on a temporal scale. From a spatial point of view, large structural deflections induce misalignments which determine the local solution of contacting tooth surfaces with moving contact points, involving geometric and contact nonlinearities. From a temporal point of view, gears generate small-amplitude high-frequency vibrations which are affected by significantly larger motions of the supporting structure (e.g. eccentricities or global driveline torsional resonances). This requires that the full system dynamics are solved using considerably small timestep and solution tolerances. Given the required mesh refinement, non-linear Finite Element simulations currently yield impractical computational effort and cannot be used for dynamic simulations [10]. Specific methodologies with different complexity have been developed for gearbox dynamics, like semi-empirical [11,12], analytical [13], FE [14], multibody [15,16], and hybrid models [17-19], however, no general technique is currently available.

This background of uncertainty makes gearbox testing of crucial importance for a successful design.

Severe challenges are present also from a testing point of view. Gear whine vibrations are in fact extremely small but at the same time powerful: tooth deflections and microgeometry modifications are in the order of $10^{-6} \mathrm{~m}$ [6], however they act on a gear mesh stiffness which is in the order of $10^{8}-10^{9} \mathrm{~N} / \mathrm{m}$. Such meshing vibrations convert into shaft angular vibrations in the order of $10^{-4} \mathrm{deg}$. Although very limited experimental measurements are available, as reported in the next subsection, gear TE is considered to be directly related to gear meshing vibrations; the related reasons are analysed in Section 2.

\subsection{Literature survey on transmission error measurement}

TE is traditionally considered to be a metric for gearbox $\mathrm{NVH}$, since it is related to varying displacements at the tooth mesh and triggers oscillating angular motions [6]. There is however little experimental evidence of correlation towards gearbox noise or gearbox case vibrations [20], limited availability of experimental data and incomplete discussion of measurement methods which can be industrially viable. As discussed in this section, reasons for such lacks are related to the complexity of the measurement. In fact, TE measurements have been traditionally limited to isolated gear pairs tested under extremely light load conditions mainly to verify gear manufacturing accuracy [21]. Measurement examples on specifically designed gear test rigs are also available in literature [22-25]. Although such boundary conditions are extremely different from the ones the gears are subject to in the gearbox assembly, it is possible to see how substantial information can already be drawn from single flank TE measurement [26]. More recently, attempts to extend the single flank measurement philosophy to the case of loaded gears and investigate the interactions between tooth deflections and microgeometry are being made [27]. In the same reference the main measurement challenges are explained and can be summarized into an extreme accuracy requirement, in the order of $10^{-5} \mathrm{deg}$, to be reached with non-intrusive instrumentation, which is also robust towards assembly errors. These challenges explain the rarity of TE measurements in real-life gearbox. Ref. [20] represents one of the few discussions of TE measurement on a series production manual transmission, showing how TE can be measured in quasistatic conditions by means of encoders and in dynamic conditions by means of accelerometers. A few operating conditions are discussed for a few microgeometry variants and correlation between dynamic measurements and noise is found. No discussion of instrumentation and measurement methods is provided by the paper. An additional industrial reference can be found in [28], where measurements on an assembled rear axle are performed by encoders. More controversial correlations towards noise are reported for a planetary gearbox in [29], where the TE is measured in a non-intrusive way by laser vibrometry and seems to have little influence on emitted noise. The need for industrially viable measurement methods is clearly outlined in a related work [30], along with a description of main ones and related instrumentation.

Concerning instrumentation for TE measurement, three main classes can be identified: optical rotary encoders, rotational laser vibrometers and paired linear accelerometers.

High-accuracy analogue encoders and low-cost digital encoders can be distinguished within optical rotary encoders. The most established way of measuring TE uses high-accuracy analogue encoders with a high number of angular divisions which typically output a sinusoidal voltage waveform. The sinusoidal waveform completes one period every angular division. Numbers of angular divisions are typically 18,000 [25] and 36,000 [24], however they can even reach 90,000 [31] and 225,000 [34]. The number of samples per rotation can be further increased by interpolating the analogue sinusoid and resolve fractions of an angular division, given the extreme accuracy of such instruments. Typical error figures are below $\pm 1 \%$ of the grating period [31] and allow placing up to 100 interpolation points within one analogue sinusoid. Therefore millions of angular divisions per rotation can be easily reached by interpolation. As pointed out in [6], it is important to analyse measurement error by Fourier analysis in order domain, where most of the error appears to be concentrated at low orders [32,33] and therefore favourably far from tooth meshing orders. High accuracy encoders can have separate scanning head and grating disk; in this case it is important that the two are accurately positioned and aligned with respect to each other [34]. To simplify mounting, integrated rotor/stator assemblies can be used: these can have a connecting shaft [32] or a connecting hub [35]; the latter improves connection stiffness and alignment [35].

More recently, low-cost digital encoders have been successfully used to measure TE [23,36,38]. These encoders have much lower numbers of angular divisions with respect to high-accuracy analogue encoders, in particular from 1800 to 4096 for the referenced articles. Measurement error amplitudes are mentioned in [6] to be surprisingly comparable to those of the high-accuracy analogue encoders; quantitative measurements are shown in [37] for a 5000 divisions analogue encoder 
which is interpolated to reach 20,000 divisions and in [38] for two equal digital encoders having 3600 divisions. A lower number of angular divisions is expected to decrease measurement accuracy [36,39].

Examples of TE measurement by laser vibrometry are limited and can be found in $[29,30,40]$. Rotational laser vibrometers measure angular velocity and provide the main advantage of a contactless measurement. Although accuracy improves with speed, the integration required to calculate TE from velocity represents an operation which can introduce errors. Practical limitations include the necessity of visual access to the shaft to be measured, which should also be oil-free. The high cost of the laser instrumentation might represent an additional practical limitation.

Linear accelerometers can be tangentially mounted to the gear bodies to measure rotational acceleration and calculate TE $[6,20,25]$. A more recent study shows how also the full field of oscillating rigid-body motion can be reconstructed [41]. Accelerometers provide significant advantages for dynamic measurements, since the transfer path between the torsional vibration source and the instrument is kept to a minimum limiting unwanted resonance effects. Furthermore, since accelerations increase with the square of the angular frequency the accuracy of the TE measurement quickly improves with speed. On the other hand, double integration of acceleration to obtain TE represents a complex operation [41] and the measurement becomes more complex from the instrumentation point of view [25], because accelerometers require precise calibration and alignment with respect to the gear bodies and the rotation axis. Furthermore signals need to be acquired from rotating components, e.g. by slip rings or by telemetry. Challenges arise in both cases for required space for mounting and balancing; additional issues for slip rings are due to electric noise introduced by contacting brushes and due to wear of the slip ring itself, while for telemetry are time synchronization of channels and supply of power.

Concerning measurement methods, a more dedicated and general review can be found in [42]. In particular, a main distinction can be made for measurement methods, which can be subdivided in timing methods and ADC methods. The traditional approach to measure TE via high-accuracy analogue encoders belongs to the ADC methods: it aims at a direct measurement of the angular position by resolving the smallest angular oscillation of interest thanks to analogue encoders yielding a high number of angular divisions. This method will be discussed in Section 3.1.1 and will be referred to as the Direct Method (DM). In the attempt of improving speed limitations a timing method has been used more recently [36], namely the Elapsed Time Method (ETM), which will be discussed in Section 3.1.2. This is an indirect measurement method, which aims at an accurate angle reconstruction relying on a high-frequency timer, provided that aliasing requirements are correctly satisfied. An additional method to measure TE is based on phase analysis via a comparator [43] or demodulation [39]. These methods will not be treated in this article.

Within the outlined background on TE measurement and given the insightfulness of this physical quantity (Section 2), the present article provides the following original contributions:

- a unitary discussion of the DM and the ETM requirements is performed in Section 3.1 based on the same reference application: a precision gear pair test rig;

- in the same section a specially designed assembly is exploited in conjunction with a calibrated high-accuracy reference to obtain a direct assessment of the measurement error of low-cost digital encoders, with its standard deviation in angle and order domain, for encoders having different numbers of angular divisions;

- limitations of the measurement methods and of the required instrumentation (high-accuracy analogue encoders and lowcost digital encoders) are thoroughly discussed in Section 3.2;

- using the precision gear pair test rig, TE measurements are obtained and compared for the DM and the ETM methods in Section 4;

- the same test rig is used in combination with a pure-involute high-precision gear pair to push the limits of ETM by reducing TE amplitudes as a function of torque and demonstrate the accuracy of the measurement chain;

- the same measurement chain is applied in Section 5 to a series production gearbox for an all-electric vehicle to measure in-situ TE under load and evaluate its repeatability; a discussion of the usefulness of this measurement is also provided.

\section{Transmission error insightfulness}

TE represents for a gear pair the difference between the ideal kinematic transmission of motion and the one achieved in reality. If gears were rigid bodies, perfectly aligned and with perfectly conjugate tooth surfaces (e.g., involute profiles), the TE would be zero and the transmission of motion could be described analytically by the gear ratio. In reality, a variety of causes introduces deviations from ideal conditions and generates a variable TE. Two main quantitative definitions of TE are adopted by the gearbox engineering community. Given for each gear the rotation angle $\theta_{i}$ and the base radius $r_{b i}$ (Fig. 1), TE can be expressed as an angle or as a linear displacement.

The angular definition is useful for torsional vibrations analysis, since it provides the relationship between the actual angular position of the driven shaft with respect to an input shaft rotation:

$$
\mathrm{TE}_{\theta}=\theta_{2}-\frac{\mathrm{r}_{\mathrm{b} 1}}{\mathrm{r}_{\mathrm{b} 2}} \theta_{1}
$$




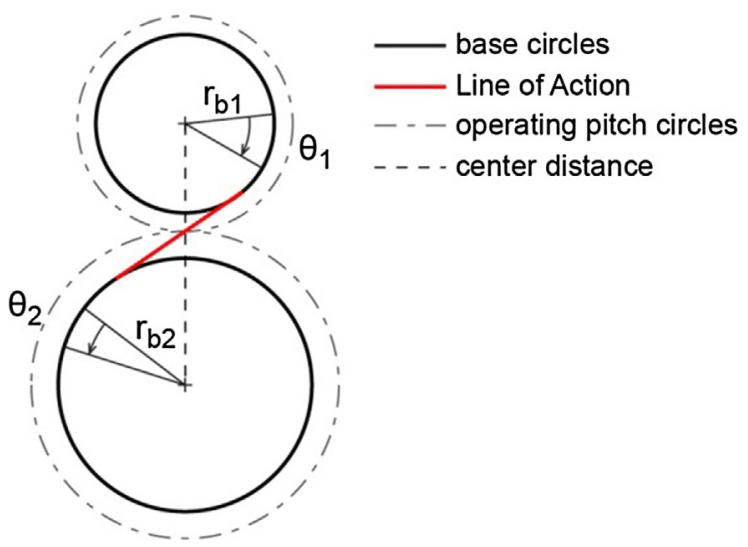

Fig. 1. Geometry for the definition of TE as a relative rotation and as a linear displacement along the Line of Action.

The linear displacement definition is related to forces exchanged by the gears, which are oriented along the Line of Action of the gear pair. This definition is usually the most useful for gear designers as it provides information on the required magnitude and shape for teeth microgeometry $[7,44]$ and is given by:

$$
T E_{L O A}=r_{b 2} \theta_{2}-r_{b 1} \theta_{1}
$$

For this reason such definition is the one most commonly used and is also adopted in the present article.

Being defined by a difference, TE provides a quantitative description of meshing vibrations in terms of relative rotations or linear displacements between the gears. TE oscillations are considered to be the cause of gear whine since they can be directly related to dynamic contact forces through the gear mesh stiffness and damping, and since they are also affected by the most relevant aspects for gear meshing and gearbox design [6,45]. A brief discussion of the main aspects related to gear mesh vibrations can be found in the following sub-sections.

\subsection{Time-varying gear mesh stiffness}

Variations of gear mesh stiffness are inherent with the process of gear meshing: for a continuous motion transmission, before one tooth pair leaves contact, a new tooth pair must have already entered into contact to take over. This process involves transfer and redistribution of the total contact load among the teeth, causing a varying stiffness dependent on the number of teeth instantaneously in contact (Fig. 2). The induced oscillations in the total elastic deflection of the gear mesh directly translate into a TE.

Oscillations of gear mesh stiffness were shown to be important sources of internal excitation [46-48] and of instability due to parametric resonance $[49,50]$.

An additional source of variable mesh stiffness is related to the geometry of the gear body. The presence of holes in the gear body affects the foundation stiffness of meshing teeth. Especially in the case of lightweight thin-rim gears, holes in the gear body (Fig. 3) can introduce amplitude modulation in the mesh deflection and therefore in the TE [51].

\subsection{Tooth microgeometry and surface imperfections}

Tooth microgeometry modifications are intentional deviations from the theoretical involutes, introduced on tooth surfaces by removing a few micrometres of material in the profile and/or the lead direction (Fig. 4). Usual modifications belong to a limited and well established set, as described in [52]. More complex topography is nowadays also possible [53].

These modifications are useful to reduce the negative effects that tooth deflections, geometric imperfections and misalignments have on gear meshing. In particular, profile modifications like tip and root relief are used to achieve a smooth

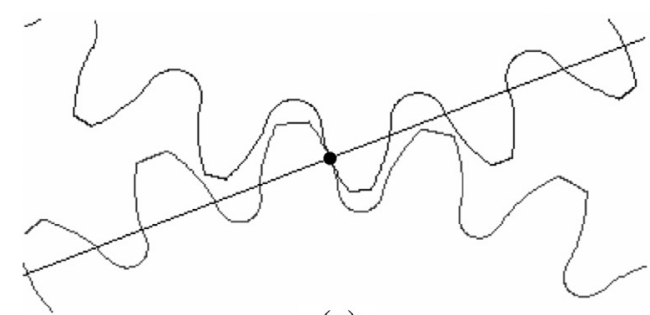

(a)

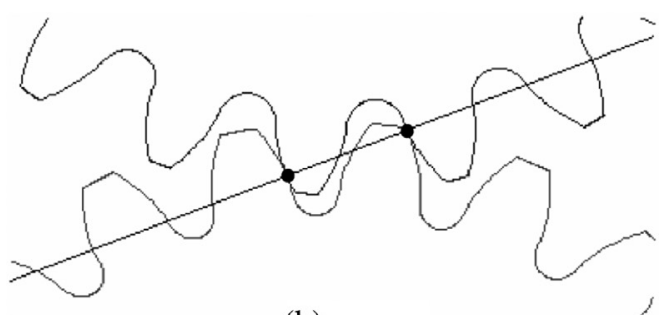

(b)

Fig. 2. Varying mesh stiffness along the mesh cycle. (a) Low stiffness: one meshing tooth pair; (b) high stiffness: two meshing tooth pairs. 


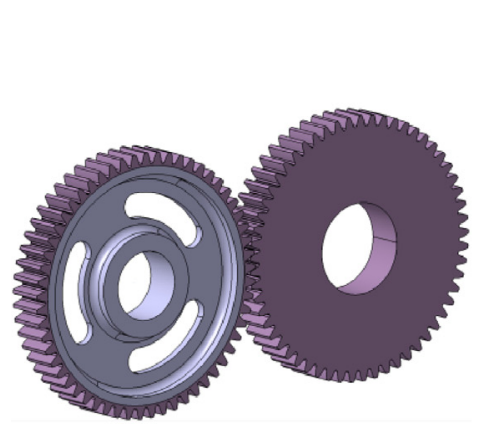

(a)

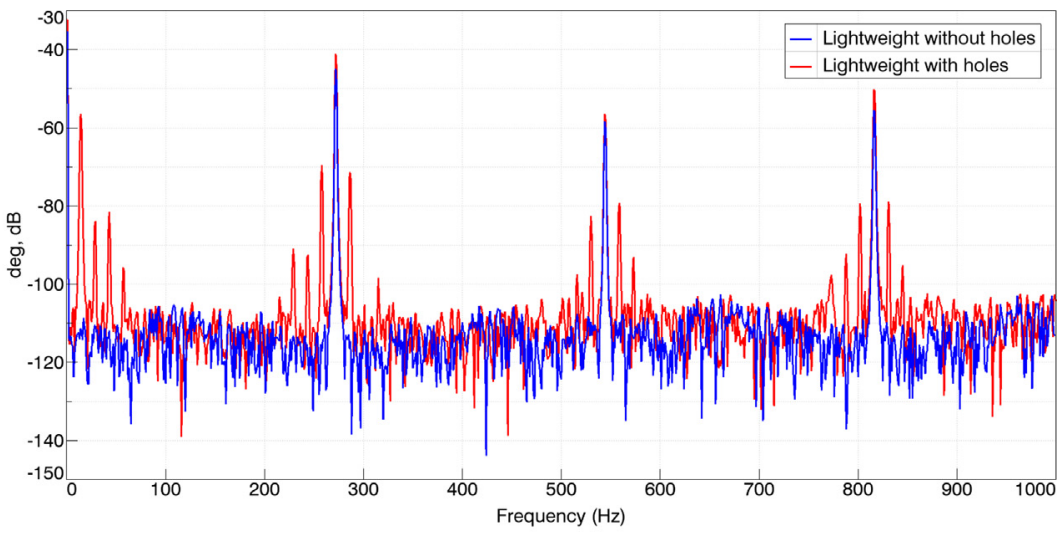

(b)

Fig. 3. (a) Lightweight gear with holes in the gear body; (b) sidebands in the calculated TE.
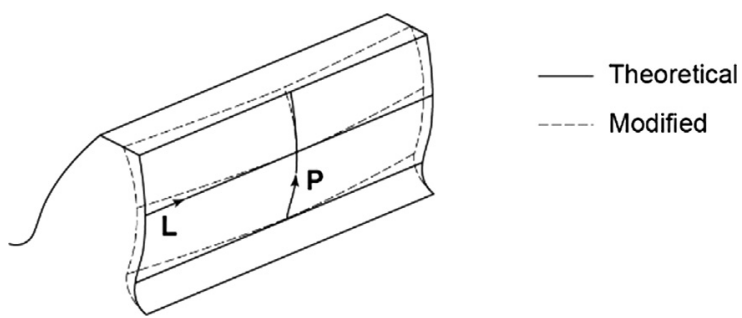

Fig. 4. Example of microgeometry modifications along the profile (P) and the lead (L) directions.

transfer of load at tooth handovers. Profile modification is optimal at a given transmitted load, by achieving minimal TE variability, and is a cause of load-dependent nonlinearity in the dynamic response of the gear pair [54]. Modifications like lead slope and lead crowning are used respectively to compensate for a fixed and a variable amount of angular misalignment between the gears [55].

Besides intentional microgeometry modifications, unavoidable deviations from ideal tooth surfaces occur due to manufacturing errors. These errors can be considered and characterized similarly to microgeometry modifications but they are usually variable from tooth to tooth [56]. A classification and description of gear manufacturing errors can be found in [57].

\subsection{Misalignments}

Gear misalignments are deviations from the nominal relative positioning of a gear pair and can be usefully distinguished in 5 components, two rotation and three displacements, on three axes: the line of action (LOA), the offline line of action (OLOA) and the axis of rotation [16].

Misalignments can be dependent or independent on loads. Load dependent misalignments are due to structural deflections. Main contributors to deflection for a gear are the shaft, the gear carrier or housing, the gear body itself and the supporting bearings. Load independent misalignments take the name of assembly errors. Main contributors to these misalignments are bearing positioning tolerances and clearances and gear axis eccentricity and alignment with respect to the actual axis of rotation. It is worth to note that misalignments induced by displaced bearing locations remain constant during gear rotation and affect TE uniformly for all the teeth, namely, in frequency domain, they only affect the amplitude and phase of the gear mesh harmonics; while misalignments induced by eccentric/tilted gear axis with respect to the rotation axis vary with shaft rotation and are instead responsible for additional harmonic content and amplitude modulation of the TE [26]. In particular, gear eccentricity introduces frequency content at sidebands which are spaced with multiples of the first order of the shaft supporting the eccentric gear; gear axis alignment introduces frequency content at sidebands which are spaced with multiples of even orders of shaft rotation.

\section{Measurement requirements and comparison between the Direct Method (DM) and the Elapsed Time Method (ETM)}

A precision gear pair test rig (Fig. 5) is used hereafter to measure TE using the DM and the ETM and to compare the requirements for each method. The test rig [58,59], available at Siemens, allows heavy instrumentation of a test gear pair under accurately known conditions of load, speed and relative positioning. In particular shafts are instrumented with 


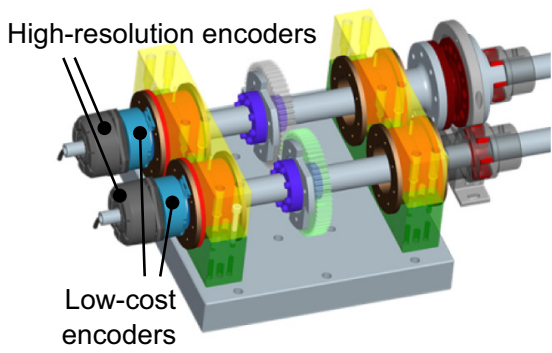

(a)

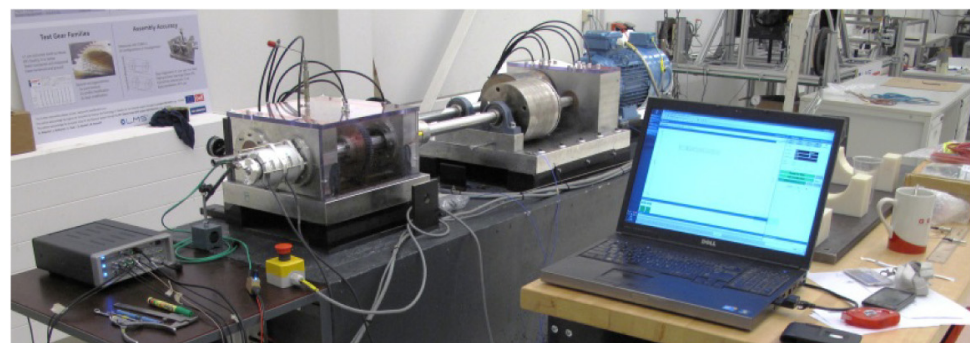

(b)

Fig. 5. Precision Gear Pair Test Rig: (a) CAD model of the test side; (b) physical installation.

traditional high-resolution analogue encoders and digital low-cost encoders. In this way both the DM and the ETM can be applied to measure TE. Through-shaft encoders with integrated bearings are preferred in order to avoid connection couplings and manual alignment between encoder scanning head and graduated disk.

Main specifications of the test rig are reported in Table 1.

A spur test gear pair in aligned conditions is analysed in this article. Test gears have the same number of teeth $Z=57$, realizing a 1:1 gear ratio, and a base radius of $r_{b}=69.631 \mathrm{~mm}$. Tooth surfaces are case hardened and precision ground to ISO 3 quality, with an overall tolerance on tooth surfaces of $\pm 1 \mu \mathrm{m}$.

Signals are acquired using an LMS SCADAS Mobile system. In particular RV4 modules are used to acquire digital signals, while V8E modules are used for analogue signals. The measurement and the data processing are performed using LMS Test. Lab Signature Testing. Main specifications of the acquisition system are reported in Table 2.

In order to proceed with the measurement it is necessary to select the encoders based on aliasing and measurement accuracy requirements. Such requirements involve evaluations of angular vibrations which must be measured by the encoders and evaluations of linear vibrations due to gear meshing. These two are related in relative terms by the TE definition (2), which can be used to convert relative displacements along the line of action of the gear pair into angles which must be measured by each encoder. Assuming that the encoders used to measure TE have similar measurement errors and that the total error must stay below $10 \%$ of the TE magnitude to be measured, $T E_{\theta}$, then the maximum error, $e_{\theta}$, allowed for each of the encoders becomes:

$$
e_{\theta}<\frac{0.1 \cdot T E_{\theta}}{r_{b 1}+r_{b 2}}
$$

Accuracy requirements can be set after an expected TE magnitude is established by preliminary simulations. In the specific case of the test rig the TE is expected to have a peak to peak value of $5 \mu \mathrm{m}$ for the operating conditions of interest. If this is considered equal to $T E_{\theta}$, we obtain:

$$
e_{\theta}<0.2 \cdot 10^{-3} \mathrm{deg}
$$

Aliasing requirements must be verified similarly for both the DM and the ETM. Such requirements can be easily set according to the Nyquist theorem by calculating the gear mesh orders or the gear mesh frequencies and considering, still from preliminary simulations, the index of the highest harmonic of interest:

$$
N_{\min }=2 s i_{\max } Z
$$

Taking a safety factor on the Nyquist lower bound $s=2$ and choosing the index of the highest harmonic of interest from the same preliminary simulations $i_{\max }=3$, the minimum number of angular divisions for each encoder becomes:

$$
N_{\min }=684
$$

It should be noted that the choice of the index $i_{\max }$ depends on the particular case to be analysed; for example it might be known that a specific system resonance is excited by a given gear order, or a criterion on the maximum allowed TE amplitude could be adopted. In any case with a different index the procedure being followed would not change, therefore the current value assumed for $i_{\max }$ will be kept in the continuation of the article.

The minimal requirements calculated above are translated with the next subsection into real specifications for the DM and the ETM, so that the two methods can be compared.

\subsection{Measurement accuracy requirements verification}

\subsubsection{Direct Method (DM)}

According to the DM, it is necessary to resolve the smallest angular vibration of interest. This can be taken as one order of magnitude smaller of the TE magnitude: 
Table 1

Main specifications of the Precision Gear Pair Test Rig.

\begin{tabular}{lll}
\hline Parameter & Range & Uncertainty \\
\hline Speed & $0-4500 \mathrm{rpm}(0-75 \mathrm{~Hz})$ & Measured \\
Torque & $0-500 \mathrm{Nm}$ & $\pm 0.05 \%$ \\
Angular misalignments & $0-2 \mathrm{mrad}$ & $0.1 \mathrm{mrad}$ \\
Parallel misalignments & $0-0.3 \mathrm{~mm}$ & $0.020 \mathrm{~mm}$ \\
\hline
\end{tabular}

Table 2

Main specifications of the acquisition system.

\begin{tabular}{lll}
\hline Acquisition mode & Maximum input rate & Time accuracy \\
\hline RV4 digital tacho input & $1 \mathrm{MHz}$ & $1.2 \mathrm{~ns}$ \\
V8E analogue input & $204.8 \mathrm{kHz}$ & - \\
\hline
\end{tabular}

$$
\Delta \theta_{\min }=0.1 \cdot T E_{\theta}
$$

Hence the required number of angular divisions, $N_{D M}$, can be calculated as:

$$
N_{D M}=\frac{2 \pi}{\Delta \theta_{\min }} \approx 880,000
$$

In order to achieve this number of angular divisions, a high-resolution analogue encoder can be used. In particular the model Heidenhain RON 285 was selected since it has a smallest recommended measuring step of $0.1 \cdot 10^{-3}$ deg which is four times smaller than $\Delta \theta_{\min }$ and two times smaller than the maximum allowed error $\mathrm{e}_{\theta}$. Furthermore the measurement error is accurately documented by means of a calibration diagram provided for each encoder by the manufacturer (see next subsection). The selected encoder outputs 18,000 sinusoidal voltage waveform periods per rotation so that further interpolation can be performed on each analogue period. In this specific case 50 intermediate points would be sufficient to reach the required number of angular divisions. An example of measured voltage waveforms is provided in Fig. 6 for one encoder channel.

Given the number of angular divisions, $N_{D M} \approx 880,000$, and the minimum number of angular divisions which is sufficient to verify aliasing requirements, $N_{\min }=684$, it is important to note that the requirement is exceeded by about 1200 times.

This represents a key limitation of the DM, since the encoder will have to generate signals with extremely high frequency already at low speed. Comparisons for our specific case are reported in Section 3.2.

\subsubsection{Elapsed Time Method (ETM)}

According to the ETM, measurement accuracy is influenced by the measurement error of each encoder and by the time accuracy of the acquisition system. In fact, the ETM allows reconstructing a continuous angle by interpolation from a discrete set of angular increments, thanks to an accurate measurement of time at which each increment happened [36]. In order to achieve sufficient time accuracy, encoders must output a digital signal which is periodic with each angular increment; a high-frequency clock can then trigger on this digital signal to assign a timestamp to each detection of a new angular increment.

Given the extremely small vibration amplitudes to be measured, it is useful to evaluate the accuracy requirements of the full measuring chain (Fig. 7). Particular care is being taken here to follow the terminology guidelines provided by the International Vocabulary of Metrology [60]. The total measurement error on a new angular increment, $E_{\Delta \theta}$, will be:

$$
E_{\Delta \theta}=\Omega\left(e_{\Delta t}+e_{r}+e_{p}\right)+e_{\Delta \theta}\left(\theta_{i}\right)
$$

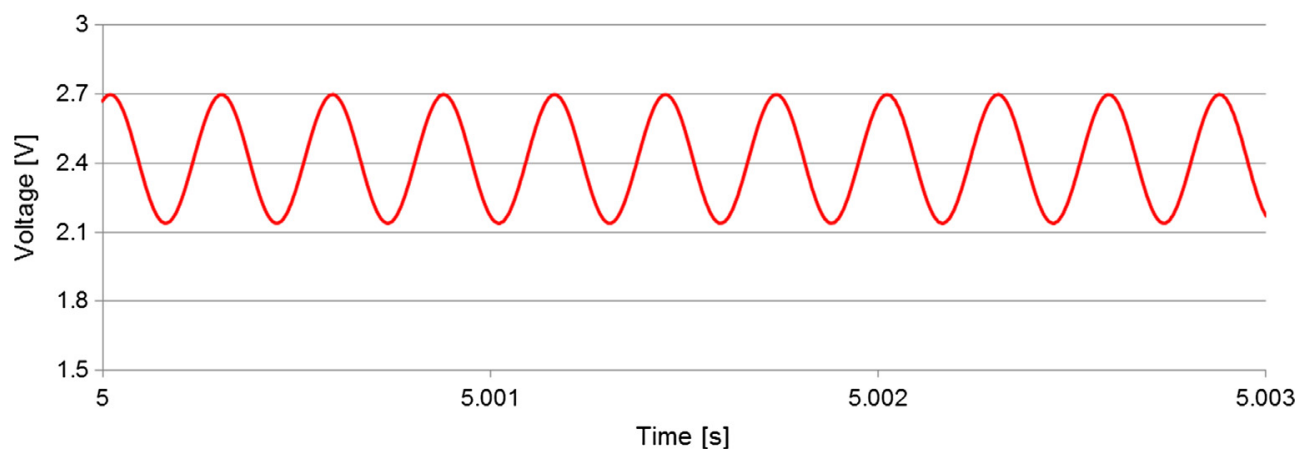

Fig. 6. Measured voltage waveform for one high-resolution analogue encoder channel. 


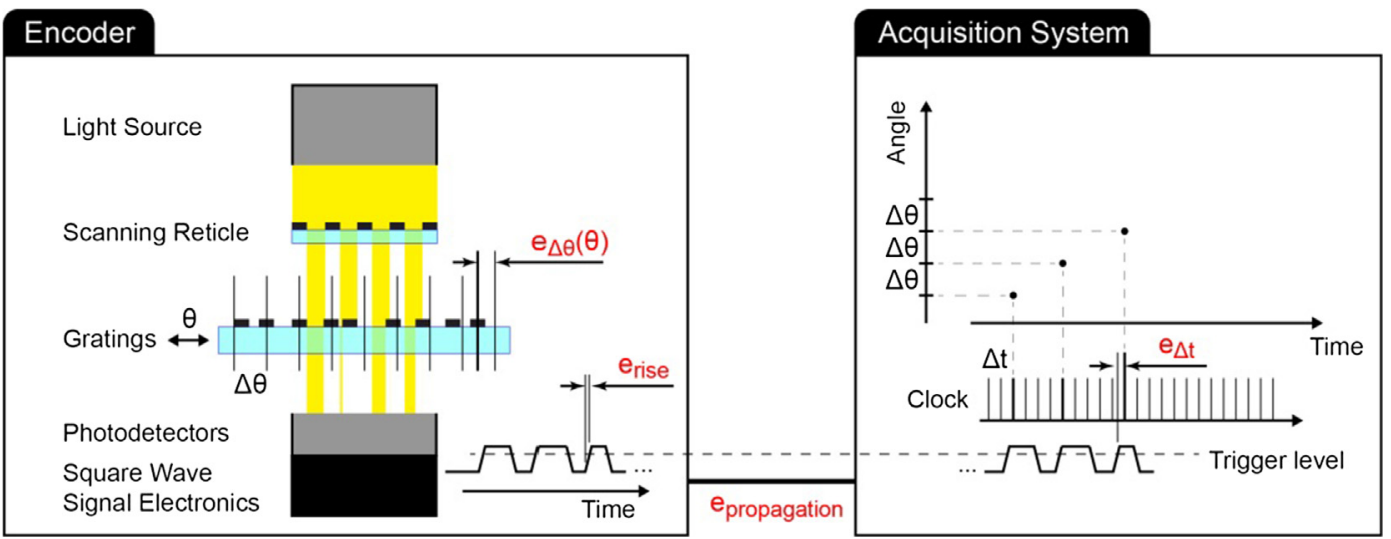

Fig. 7. Angle measuring chain for a low-cost digital encoder.

where $\Omega$ represents the instantaneous angular velocity of the measured shaft, which converts the error on time in angle units; $e_{\Delta t}$ is the timing error and is due to the finite resolution of the high-frequency clock; $e_{r}$ is the rise time error and is due to the time required for the digital square wave to reach the acquisition trigger level; $e_{p}$ is the propagation time error and is due to the cable travelling time for the signal to reach the acquisition system and $e_{\Delta \theta}\left(\theta_{i}\right)$ is the encoder grating error due to uneven spacing at each angular increment $\theta_{i}$.

Errors on time are delays in acquiring samples and become more important with linear trend as angular velocity increases. $e_{\Delta t}$ arises from the time quantisation performed by the acquisition system clock and therefore generates random error. $e_{r}$ is typically constant and depends on the performance of the digital signal electronics. $e_{p}$ is similarly constant and depends on the cable length to be travelled by the signal. While $e_{r}$ and $e_{p}$ affect angle measurement trueness, $e_{\Delta t}$ affects angle measurement precision. $e_{\Delta \theta}(\theta)$ is assumed to be mainly a source of systematic measurement error due to manufacturing errors which make the gratings unequally spaced or due to eccentricity and misalignment of the encoder graduated disk. Therefore such error also affects measurement trueness. This assumption is validated in the course of this paragraph.

Values for the above-mentioned sources of error can be found on the specifications provided by the encoder manufacturer [61] and the acquisition system manufacturer [62]. In particular, considering the Heidenhain ERN family of low-cost digital encoders and the LMS SCADAS acquisition system:

$$
\begin{aligned}
& e_{\Delta t}=1.2 \cdot 10^{-9} \mathrm{~s} \\
& e_{r}=10 \cdot 10^{-9} \mathrm{~s} \\
& e_{p}=6 \cdot 10^{-9} \mathrm{~s} \text { (per meter cable) }
\end{aligned}
$$

These values are reported in degrees at different shaft speeds in Table 3 and should be compared to (4) to assess the impact on the required measurement accuracy.

A few observations can be drawn from Table 3. All time errors are negligible at low speed. The error due to timing is two orders of magnitude below the accuracy requirement across the full speed range. Therefore the acquisition system does not introduce particular limitations to measurement accuracy. Care should be taken for errors due to rise and propagation time: encoders of the same family and having the same cables length must be used, so that the related errors are very similar, and since TE is calculated by the angles difference such errors cancel out.

No information is available on the encoder grating error as the encoder manufacturer does not provide calibration diagrams for low-cost encoders. For that reason, the encoder grating error has been measured for different encoder types having different number of angular divisions above the Nyquist requirement (Table 4).

The grating error for the low-cost digital encoders has been measured by taking a high-resolution analogue encoder as reference angular position.

Table 3

Low-cost digital encoder (ERN types) errors for different shaft speeds.

\begin{tabular}{lllll}
\hline $\begin{array}{l}\text { Shaft speed } \\
{[\mathrm{rpm}]}\end{array}$ & $\begin{array}{l}\text { Encoder grating error } e_{\Delta \theta} \\
{[\mathrm{deg}]}\end{array}$ & Rise time error $\Omega e_{r}[\mathrm{deg}]$ & $\begin{array}{l}\text { Propagation time error } \Omega e_{p}[\mathrm{deg} / 1 \mathrm{~m} \\
\text { cable] }\end{array}$ & $\begin{array}{l}\text { Timing error } \Omega e_{\Delta t} \\
{[\mathrm{deg}]}\end{array}$ \\
\hline 10 & - & $+0.55 \mathrm{E}-6$ & $+0.36 \mathrm{E}-6$ & 0 to $0.72 \mathrm{E}-6$ \\
2450 & & $+0.15 \mathrm{E}-3$ & $+0.09 \mathrm{E}-3$ & 0 to $0.017 \mathrm{E}-3$ \\
4500 & $+0.27 \mathrm{E}-3$ & $+0.16 \mathrm{E}-3$ & 0 to $0.033 \mathrm{E}-3$ \\
\hline
\end{tabular}


Table 4

Low-cost digital encoders: analysed types and number of angular divisions.

\begin{tabular}{ll}
\hline Encoder type & Number of angular divisions \\
\hline ERN 120 & 5000 \\
ERN 420 & 5000 \\
& 2048 \\
& 1024 \\
\hline
\end{tabular}

The results of such measurement is graphed in Fig. 8 along two shaft rotations for the ERN 120 encoder against the calibration chart of the high-resolution analogue encoder. Results appear to be extremely repeatable, as highlighted by tight 95\% confidence bounds calculated for the low-cost digital encoder over 20 full rotations. The low-cost digital encoder has an overall accuracy of $3 \cdot 10^{-3} \mathrm{deg}$, while the high-resolution analogue encoder has an overall accuracy of $1.32 \cdot 10^{-4}$ deg. This might lead us to the conclusion that the high-resolution encoder is therefore above ten times more accurate with respect to the low cost digital encoder.

However, as pointed out in [6], it is important to analyse the order content of the measurement error rather than its overall peak to peak value. Fig. 9 shows a comparison in this sense between the low-cost digital encoder and the high-resolution analogue encoder. Results show how the measurement error, both for high-resolution and low-cost encoders is concentrated at the first few shaft orders. The high-resolution encoder remains substantially more accurate than the low-cost digital encoder, however after order 20 the error amplitude stays below $1 \cdot 10^{-5} \mathrm{deg}$ for both encoders. Since gear meshing orders are typically above order 20 , the encoder grating error for the digital low-cost encoder can be considered equal to the same value $e_{\Delta \theta}=1 \cdot 10^{-5} \mathrm{deg}$.

A similar analysis can be repeated for the ERN 420 encoders to observe how measurement error depends on the number of angular divisions. Measurements are still extremely repeatable; this time standard deviation is calculated after performing the Fourier Transform to order domain for each shaft rotation. Best-fit curves are also obtained after selecting an exponential model as in (13), where 0 represent the order number, and using the coefficients in Table 5. Results are shown in Fig. 10 and show similar behaviour and values as in Fig. 9. A standard deviation below the average value of the error confirms the systematic nature of the error across the full order range. A comparison between best-fit models is reported in Fig. 11 and highlights equivalent performance for the encoders having 5000 and 2048 angular divisions and a slightly higher error for the one with 1024. This allows drawing the conclusion that a higher number of angular divisions results in a limited improvement on measurement accuracy.

$$
\log _{10}\left[e_{\Delta \theta}(\mathrm{O})\right]=a_{1}+a_{2} \exp \left(-a_{3} \mathrm{O}\right)+a_{4} \exp \left(-a_{5} \mathrm{O}\right)
$$

As a conclusion of this subsection, a graphic summary to compare all the discussed errors (Table 3, Fig. 9) for all shaft speeds is presented in Fig. 12. All error components are at least ten times below the smallest angular vibration of interest $\Delta \theta_{\min }$ if care is taken to ensure cancellation of rise time and propagation time errors. Therefore digital encoders are suitable to accurately measure TE.

\subsection{TE measurement limitations}

\subsubsection{Maximum angular speed}

The maximum angular speed at which TE can be measured is limited by the lowest among the following:

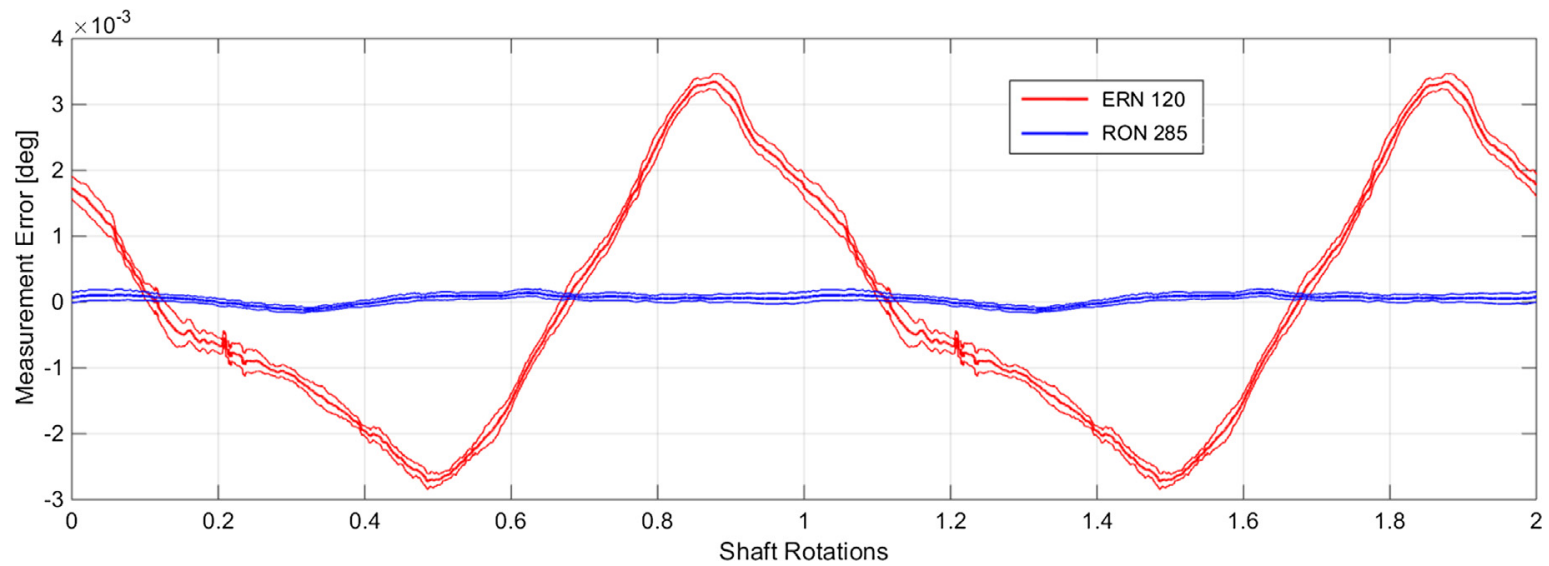

Fig. 8. Measurement error along two shaft rotations in angle domain for one high-resolution analogue RON 285 encoder and one low-cost digital ERN 120 encoder. 


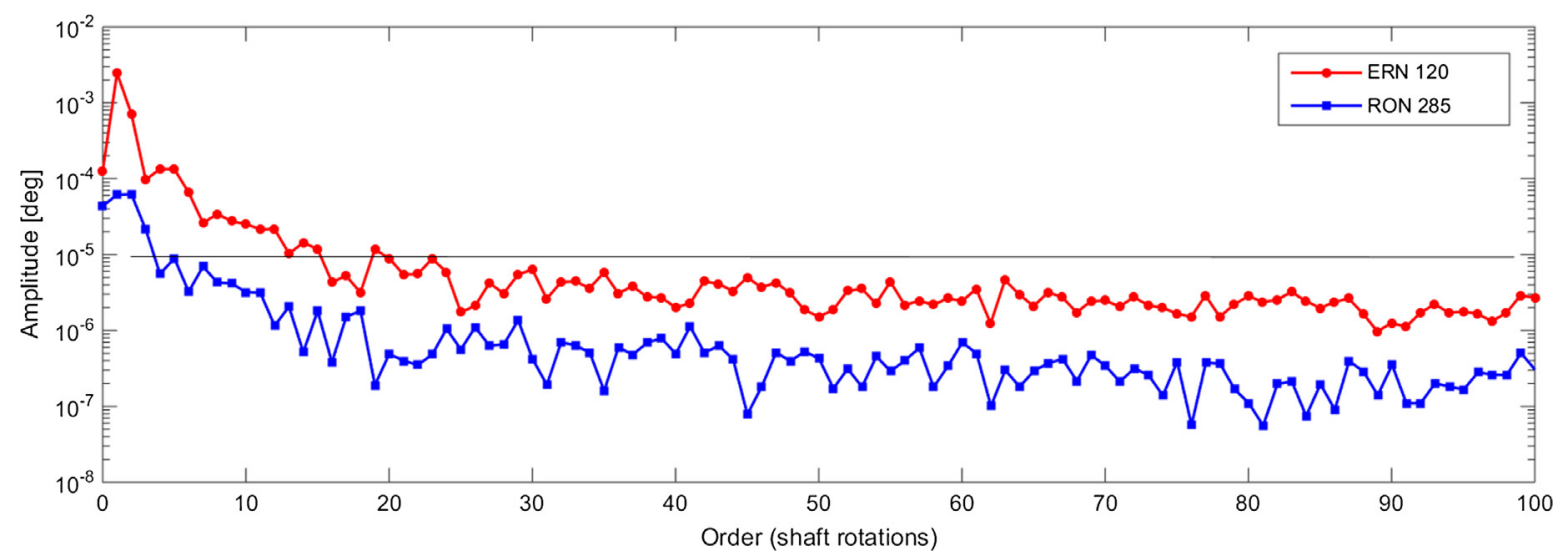

Fig. 9. Fourier Transform in order domain of the measurement error for the same high-resolution analogue RON 285 encoder and low-cost digital ERN 120 encoder.

Table 5

Best-fit model coefficients for the encoder error amplitudes in order domain.

\begin{tabular}{llll}
\hline Model coefficients & \multicolumn{3}{l}{ Fourier Transform } \\
\cline { 2 - 4 } & 1024 & 2048 & 5000 \\
\hline$a_{1}$ & -8.274 & -5.784 & -6.093 \\
$a_{2}$ & 2.517 & 2.903 & 1.972 \\
$a_{3}$ & 0.311 & 0.614 & 0.288 \\
$a_{4}$ & 3.553 & 1.540 & 1.493 \\
$a_{5}$ & 0.003 & 0.050 & 0.020 \\
\hline
\end{tabular}

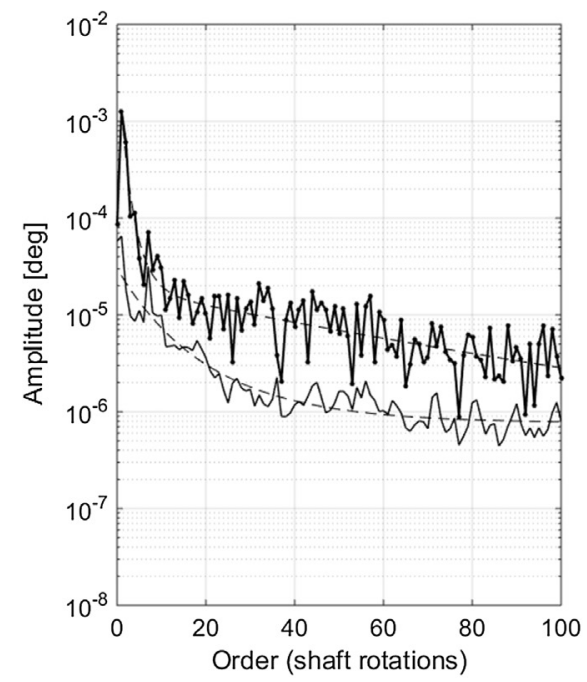

(a)

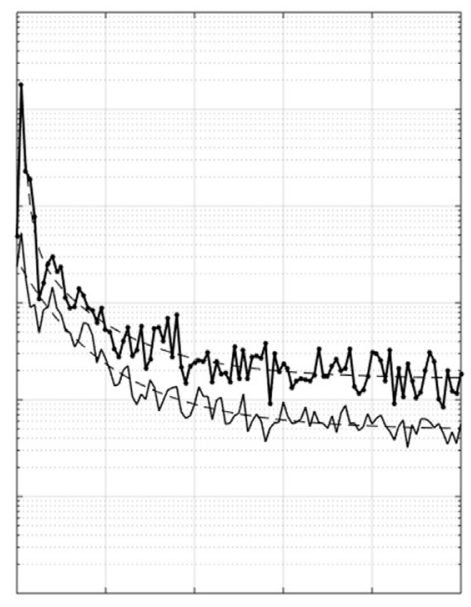

(b)

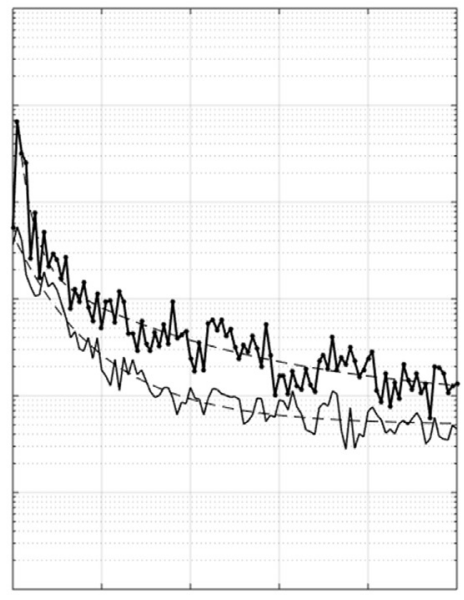

(c)

Fig. 10. Fourier Transform in order domain of the measurement error and the related standard deviation for different low-cost digital ERN 420 encoders: (a) 5000, (b) 2048, (c) 1024 angular divisions. Dashed lines represent best-fit models.

- Mechanical permissible speed

- Speed at which the photocell bandwidth is saturated

- Speed at which square wave edges are separated (only for digital encoders)

- Speed at which the acquisition system input rate is saturated

The mechanical permissible speed is defined as the speed at which the encoder can run to have infinite fatigue life. Such speed is reported by the encoder manufacturer and is mainly determined by the bearings of the encoder. When the 


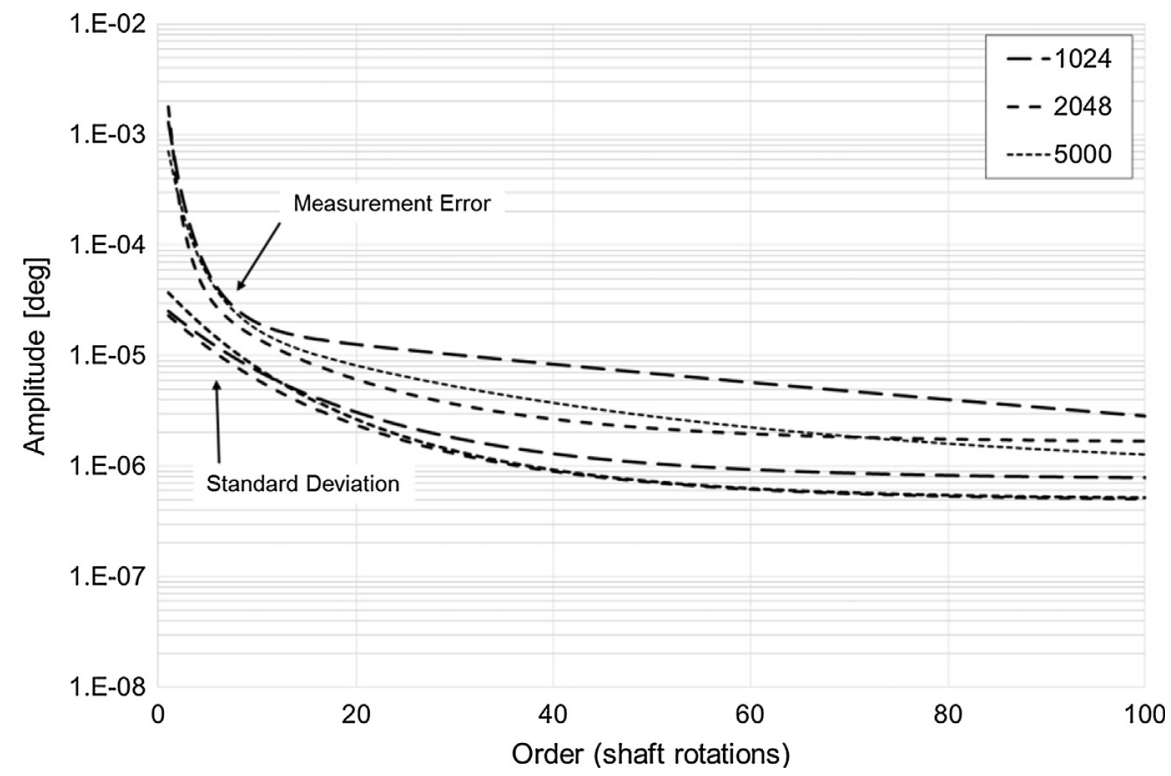

Fig. 11. Comparison of the best-fit models for measurement error amplitudes and standard deviations in order domain of different low-cost digital ERN 420 encoders: (a) 5000, (b) 2048, (c) 1024 angular divisions.

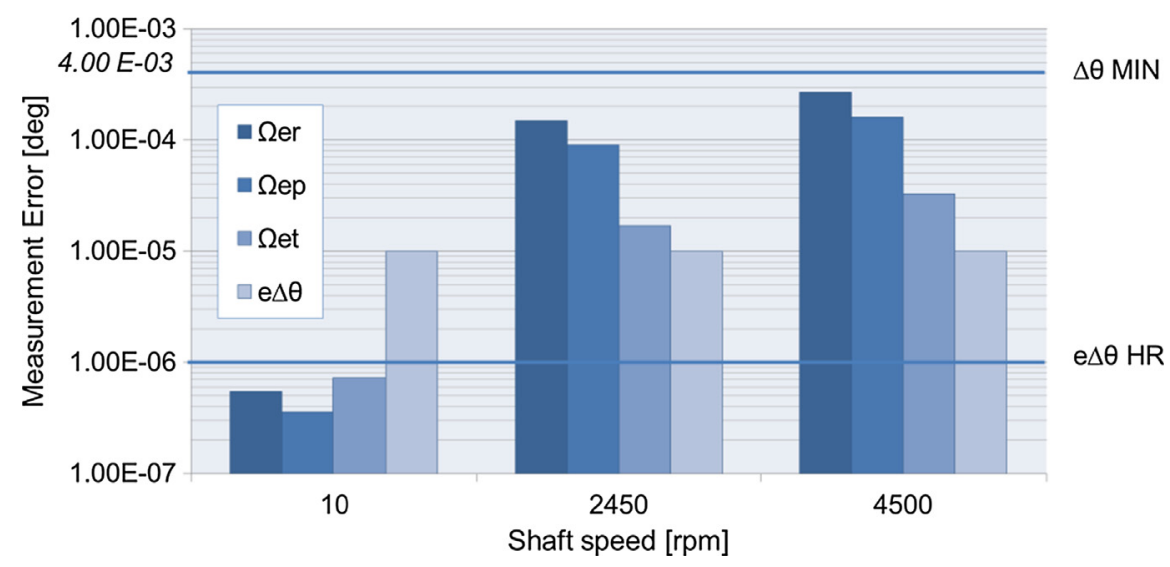

Fig. 12. Summary of all the analysed encoder errors for different shaft speeds.

mechanical permissible speed is exceeded the bearings start accumulating damage. Therefore, if needed, this speed can be exceeded at the cost of replacing the encoder when deterioration on the measurement becomes noticeable.

All the remaining speed limitations are due to electronics. A photocell together with related electronic circuitry is used to detect light by the imaging scanning principle and to generate either the analogue or the digital waveform. Such electronic system can generate a synchronous waveform with the passage of the encoder gratings within a finite bandwidth. The bandwidth at which the electronics attenuate the output waveform of $-3 \mathrm{~dB}$ in amplitude is equal to $f_{e}=300 \mathrm{KHz}$ for ERN encoders and to $f_{e}=180 \mathrm{KHz}$ for RON encoders. The angular speed in rpm at which this limitation is reached can be calculated as:

$$
n_{e}=\frac{60 f_{e}}{N}
$$

It is worth mentioning that above this frequency the output signal would reduce its amplitude and would have an increasing phase delay, however in case of need such bandwidth can be extended by measuring the frequency response function of the electronics.

Furthermore, for digital signals, the electronic system is able to generate a square wave with a given minimum time between two subsequent edges of a square wave cycle (edge separation time). The edge separation time for digital encoders belonging to the ERN family is $t_{s}=0.39 \cdot 10^{-6} \mathrm{~s}$. The angular speed in rpm at which this limitation is reached can be calculated as: 


$$
n_{s}=\frac{60}{t_{s} \cdot 2 N}
$$

Finally, at a given angular speed, the acquisition system must be able to acquire the waveform generated by the encoders. In case of analogue signals, the maximum input rate allowed by the acquisition system is $f_{a}=204.8 \mathrm{KHz}$ for analogue signals (sampling frequency) and $f_{a}=1 \mathrm{MHz}$ for digital signals (pulse rate). It should be noted that the maximum pulse rate of 1 $\mathrm{MHz}$ can be reached using the pulse skip functionality of the RV4 module [63]. Encoder pulses can in fact be skipped while still aliasing requirements are verified, namely until the actual number of pulses per rotation used stays above (6) requirement aliasing. However until $204.8 \mathrm{kHz}$ it is not needed to skip pulses and the decision is fully dependent on engineering judgement. The angular speed at which the acquisition is reached can be calculated as:

$$
n_{a}=\frac{60 f_{a}}{N}
$$

Speed values for all the analysed encoders are compared in Table 6 with the first limiting speed highlighted in bold. A clearer graphic representation of the same data is reported in Fig. 13.

Table 6 and Fig. 13 show one of the main advantages of the ETM over the DM: the maximum possible angular speed for the ETM is at least in the order of a hundred times higher with respect to the DM. The table also shows how limitations due to the response of the photocell are reached well before the mechanical and the acquisition system limits. In fact, only for the high-resolution analogue encoder the acquisition system limits the maximum speed to $14 \mathrm{rpm}$. Even if this limitation was not present, the photocell would limit the maximum speed to only $600 \mathrm{rpm}$. Therefore, given the specifications reported in Table 6, it is possible to say that speed limitations are set by the encoders. Actually the mechanical permissible speed represents the speed limitation only in the case of the encoder with the lowest number of angular divisions. In fact, speed limitations due to electronics are inversely proportional to the number of angular divisions of the encoder. Given that the error is weakly dependent on the number of angular divisions (Fig. 11), if photocell saturation becomes a limiting factor, it is advisable to select the first available number of angular divisions above the aliasing requirement.

\subsubsection{Required mounting tolerances}

The second key measurement limitation stems from the mounting tolerances required by each encoder type. In particular high-resolution analogue encoders require tighter alignment tolerances which are usually difficult to achieve in industrial environment (Fig. 14). These tolerances are needed to ensure that the absolute encoder error is kept to a minimum. Since most of this error is concentrated at low orders (Figs. 8 and 9) and TE accuracy requirements are less strict at low orders, a higher error can be tolerated for the sake of mounting simplifications. In fact for the low-cost encoders it is not necessary to guarantee alignment between encoder stator and rotor. This is possible because the stator of the low-cost encoders is kept aligned with respect to the shaft by an integrated high-precision double-row ball bearing. Mounting can thus be simplified thanks to a mounting system which is torsionally stiff but compliant in out of plane rotations.

In the case of the present gear test rig, a floating mounting system which is insensitive to shaft misalignment has been devised to keep the high-resolution encoder aligned. A positioning element has been manufactured as shown in Fig. 15. In particular, the inner bores of the encoders are normally aligned on the shaft easily, while the stator of the high-resolution encoder is aligned with respect to the stator of the low-cost digital encoder. Thanks to the high-precision bearing, the stator of the low-cost encoder has been taken as a reference after coordinate machine measurement. Using the positioning element, the high-accuracy encoder becomes insensitive to misalignment.

\section{Measurement results}

The discussion carried out in the previous section is now applied to measure TE for two different gear pairs, mounted one at a time on the described precision gear pair test rig. Measurements have been performed at low speed ( $\sim 10 \mathrm{rpm})$ and simultaneously by the DM, using high-resolution analogue RON 285 encoders, and by the ETM, using low-cost digital

Table 6

\begin{tabular}{|c|c|c|c|c|c|c|c|}
\hline \multirow[b]{2}{*}{$\begin{array}{l}\text { Encoder } \\
\text { type }\end{array}$} & \multirow[b]{2}{*}{$\begin{array}{l}\text { Angular } \\
\text { divisions }\end{array}$} & \multirow[b]{2}{*}{$\begin{array}{l}\text { Interpolated } \\
\text { angular } \\
\text { divisions }\end{array}$} & \multirow[b]{2}{*}{$\begin{array}{l}\text { Ratio with } \\
\text { Nyquist } \\
\text { requirement }\end{array}$} & \multicolumn{3}{|c|}{ Encoders performance } & \multirow{2}{*}{$\begin{array}{l}\text { Acquisition } \\
\text { system } \\
\text { performance } \\
\text { Input rate } \\
\text { saturation speed } \\
\text { [rpm] }\end{array}$} \\
\hline & & & & $\begin{array}{l}\text { Mechanical } \\
\text { permissible speed } \\
{[\mathrm{rpm}]}\end{array}$ & $\begin{array}{l}\text { Minimum edge } \\
\text { separation speed } \\
{[\mathrm{rpm}]}\end{array}$ & $\begin{array}{l}\text { Photocell } \\
\text { saturation speed } \\
{[\mathrm{rpm}]}\end{array}$ & \\
\hline RON 285 & 18,000 & $\sim 880,000$ & 1200 & 3000 & - & 600 & 14 \\
\hline ERN 120 & 5000 & - & 7.3 & 6000 & 15,400 & 3600 & 12,000 \\
\hline \multirow[t]{3}{*}{ ERN 420} & 5000 & - & 7.3 & 12,000 & 15,400 & 3600 & 12,000 \\
\hline & 2048 & - & 3.0 & 12,000 & 37,550 & 8790 & 14,650 \\
\hline & 1024 & - & 1.5 & 12,000 & 75,000 & 17,580 & 14,650 \\
\hline
\end{tabular}

Speed limitations for all the analysed encoders. 


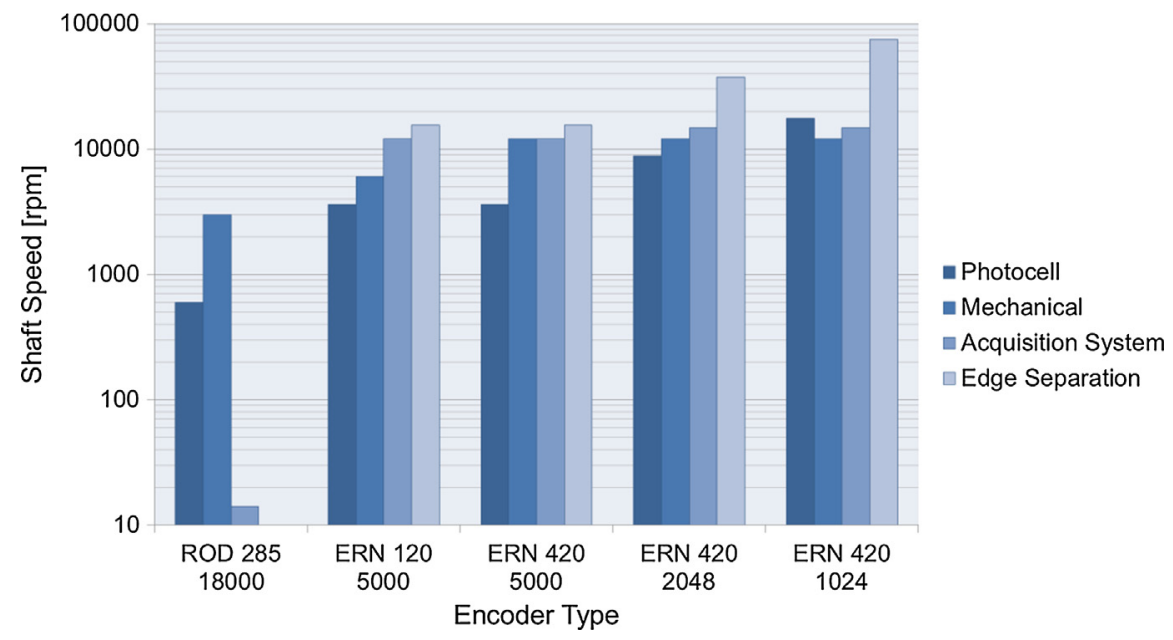

Fig. 13. Speed limitations for all the analysed encoders: graphical comparison.

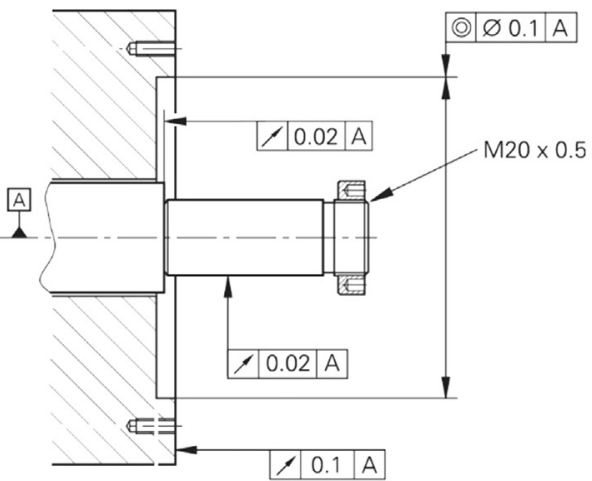

(a)

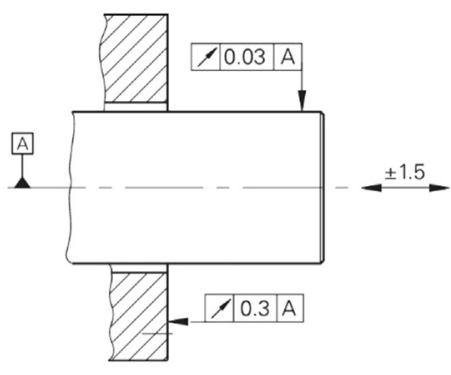

(b)

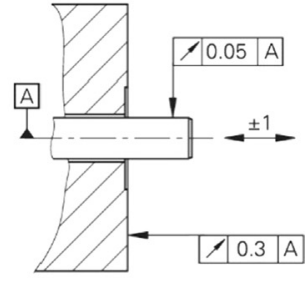

(c)

Fig. 14. Main characteristics of tolerance schemes for the high-resolution analogue encoder RON 285 (a) and for the low-cost digital encoders ERN 120 (b) and ERN 420 (c).

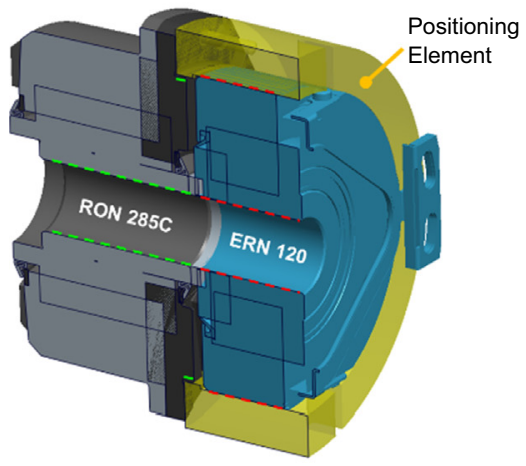

(a)

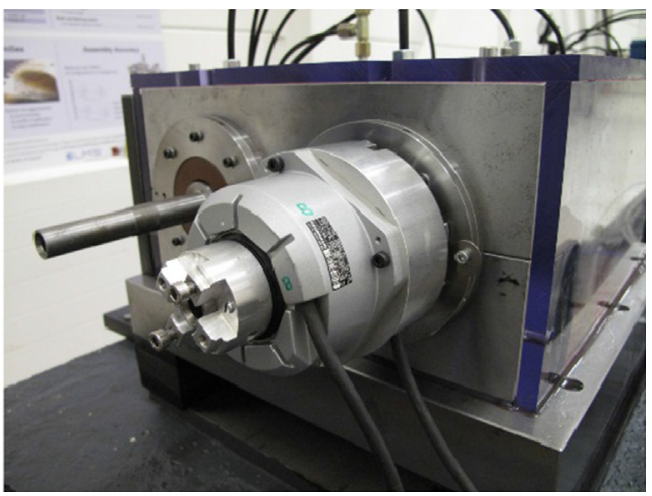

(b)

Fig. 15. (a) Encoders positioning element and positioning surfaces (indicated by dashed lines); (b) Encoders installation.

ERN 120 encoders. The TE will convert into a dynamic excitation when gears are spin at higher speed including system resonance and lubrication effects, which are not discussed in this paper. 
The first gear pair has industrial quality helical gears (ISO 7); each gear has 64 teeth to achieve 1:1 ratio. This gear pair will be used to discuss general characteristics of gear TE and to verify the overall difference between the DM and the ETM. The second gear pair is a high-precision spur gear pair (ISO 3) with unmodified tooth surfaces, namely with pure involutes. Each gear has 57 teeth, still to achieve 1:1 ratio. This gear pair will be used to understand the practical accuracy limits of the ETM, namely to answer the question: what happens if the TE lies actually below the amplitude assumed after simulations? In particular, since tooth surfaces of this second gear pair are pure involutes, TE is expected to tend towards zero if the transmitted torque is also reduced towards zero. TE analysis will therefore be performed varying the transmitted torque and observing trends for different gear meshing orders.

\subsection{Industrial quality gear pair}

Gear TE measured at a transmitted torque of $200 \mathrm{Nm}$ is reported in Fig. 16 for the DM and for the ETM across two full shaft rotations. The first important observation is that the results obtained using each method are overlapping, confirming that the low-cost digital encoders can be used in combination with the ETM to obtain accurate TE measurements.

Furthermore two expected characteristics of TE [64] can be observed (as discussed in Sections 1 and 2): the signal can be decomposed into a high-amplitude long wave component and a low-amplitude short wave component. These two components are shown separately in Fig. 17 by filtering below and above order 5, the operation is discussed without loss of generality for the ETM measurement case. The long wave has practically a purely sinusoidal trend with a fundamental period equal to the shaft rotation, highlighting that it is due to gear eccentricity with respect to the shaft axis of rotation. The absence of vibrations periodic with second shaft order highlights that the gear axis is well aligned with the shaft axis of rotation. The short wave component has a much smaller amplitude and completes 64 cycles per shaft rotation, one cycle per tooth. Irregularities on the shape of each tooth period are due to manufacturing errors.

Similar observations can be made more accurately looking at the order content of the TE (Fig. 18). The same figure also shows the order content of the composite encoder error, obtained by the sum of the two low-resolution digital encoders phased with respect to the same arbitrary angular position reference pulse. The angular error made by the encoders is converted into TE units using (4).

Concerning the encoders, it can be observed how the composite error stays well below a TE of hundredth of a micron at gear mesh orders, highlighting once again the high accuracy of the measurement.

The long wave TE component shows dominant eccentricity (shaft order 1) and very limited misalignment (shaft order 2) contributions (Table 7) with a first order with amplitude of $76 \mu$ mfollowed by a second order which is about 20 times smaller. High TE amplitudes are typical at low orders and this explains why higher encoder errors can be tolerated there.

The short wave TE component is mostly dominated by the first tooth meshing order (Table 7), followed by the higher meshing orders and limited sideband activity about 10 times smaller.

\subsection{High-precision pure-involute gear pair}

As stated, the availability of unmodified precision spur gears allows us not to push the limits of the measurement method. Since teeth of this new gear pair are perfectly conjugate, TE is expected to converge to zero when torque is reduced. Measurement are now performed relying only on the ETM and measurement and accuracy is verified by looking at TE trends with respect to the transmitted torque. A long wave TE amplitude standing below $10 \mu \mathrm{m}$ highlights extremely low eccentricity and misalignment, therefore it will be directly filtered from the TE signal. Furthermore the extreme accuracy of tooth

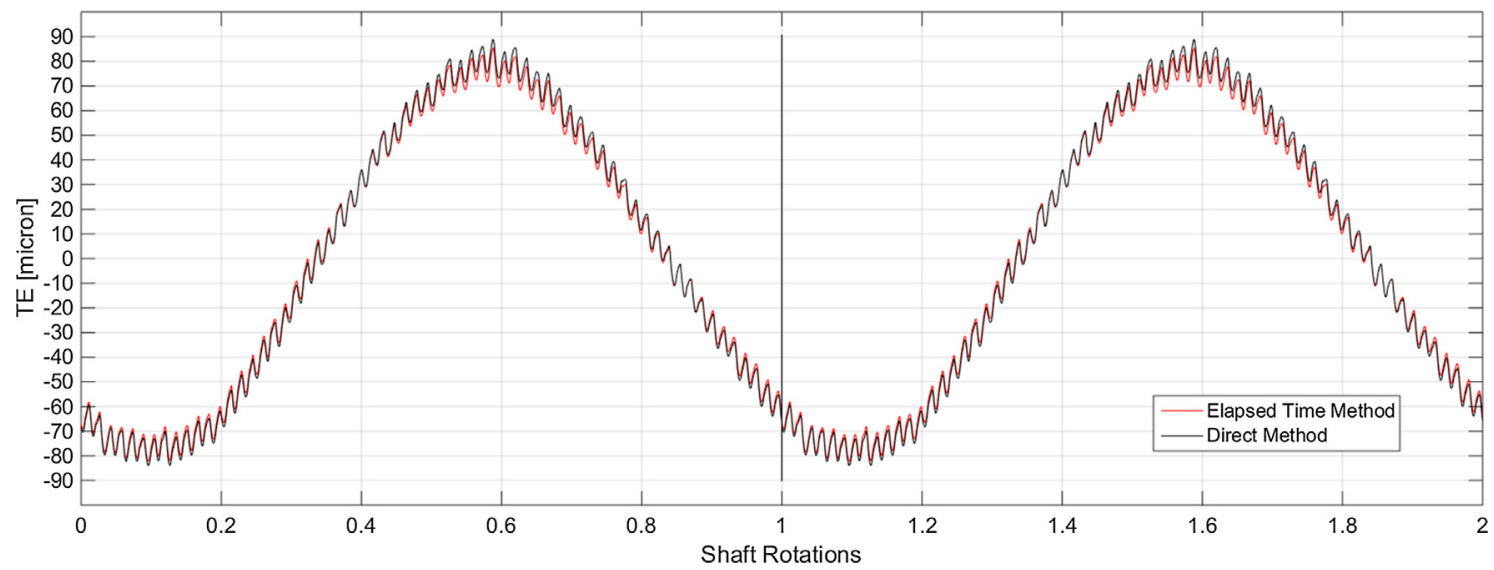

Fig. 16. Measured TE in angle domain for two shaft rotations: comparison between the DM using high-resolution analogue encoders and the ETM using low-cost digital encoders. 


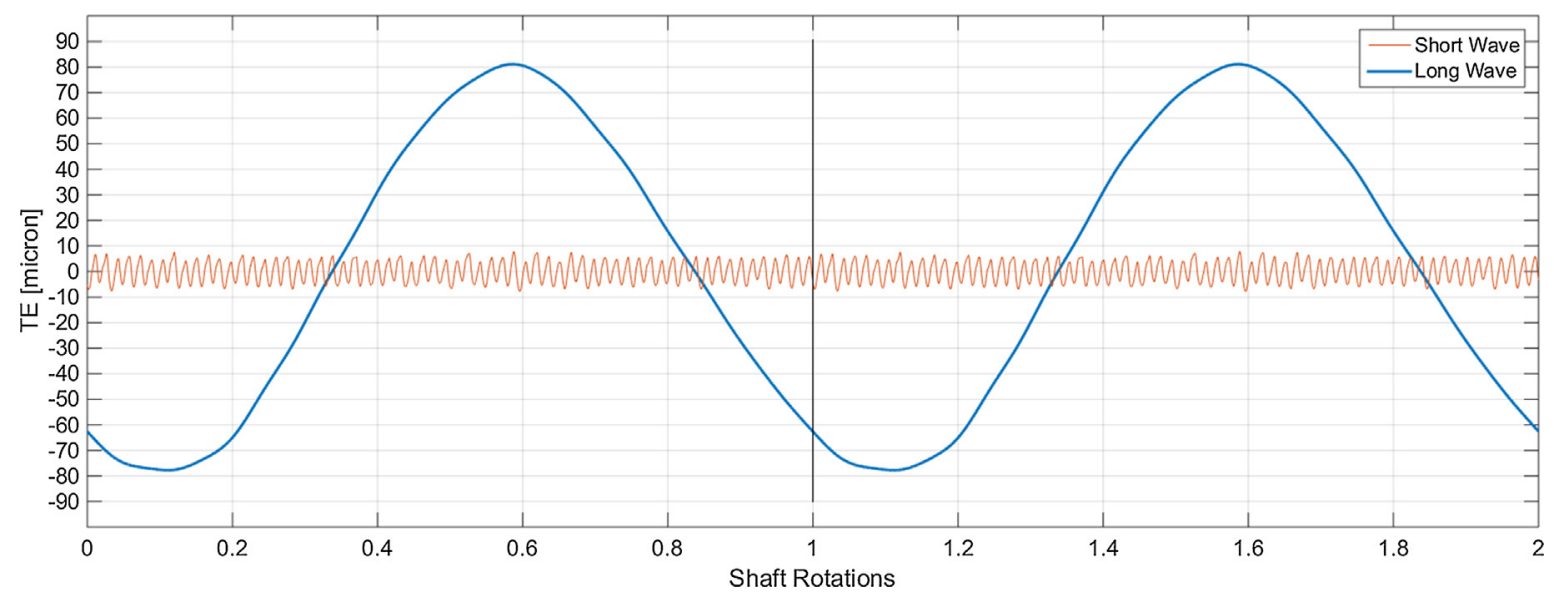

Fig. 17. Decomposition of the measured TE signal into the long and the short wave components.

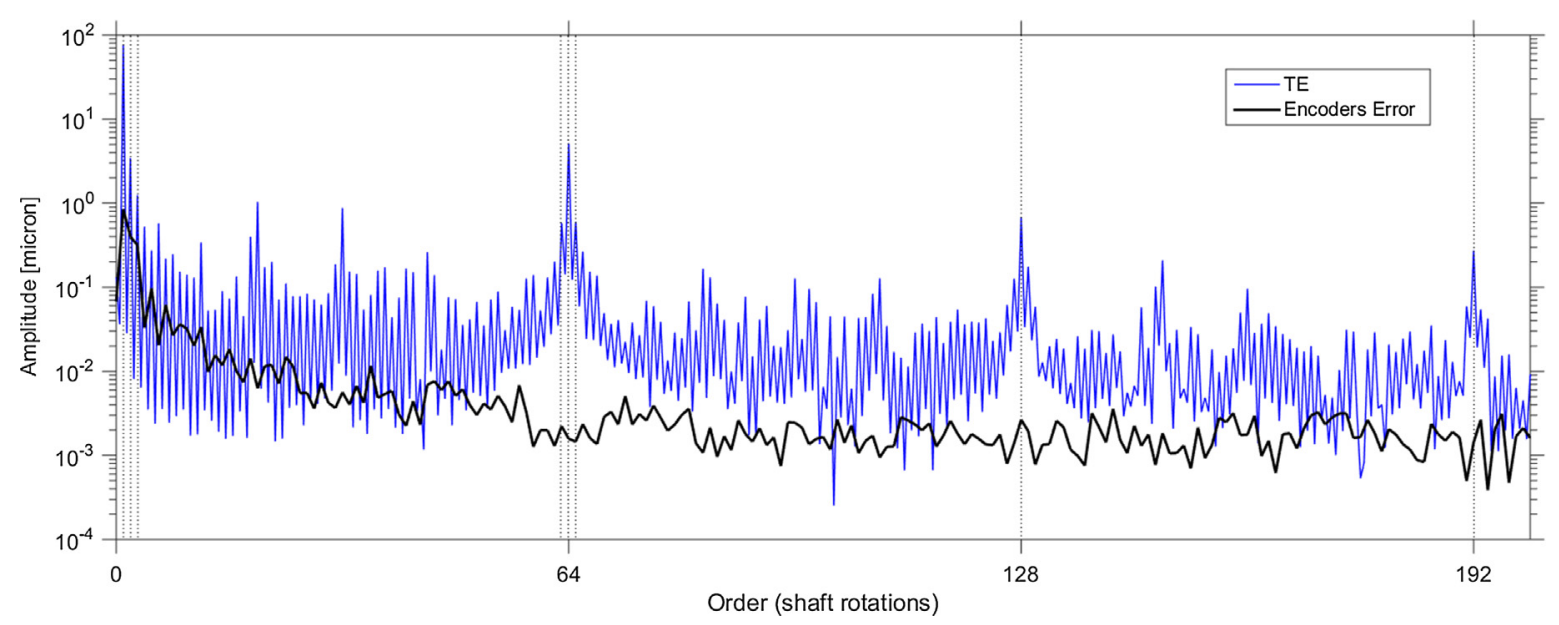

Fig. 18. Fourier Transform in order domain of the measured TE and comparison with the composite error of the low-cost encoders converted in micron.

Table 7

Comparison of the most important order components of the measured TE signal.

\begin{tabular}{|c|c|c|c|c|c|c|c|c|}
\hline \multicolumn{3}{|c|}{ Shaft orders } & \multicolumn{3}{|c|}{ Mesh orders } & \multicolumn{3}{|c|}{ Sidebands around first mesh order } \\
\hline Order & TE amplitude $[\mu \mathrm{m}]$ & Ratio w.r.t. order 1 & Order & TE amplitude $[\mu \mathrm{m}]$ & $\begin{array}{l}\text { Ratio w.r.t. } \\
\text { order } 64\end{array}$ & Order & TE Amplitude [ $\mu \mathrm{m}]$ & Ratio w.r.t. order 64 \\
\hline 1 & 75.880 & $100 \%$ & 64 & 5.178 & $100 \%$ & $64-1$ & 0.580 & $11.2 \%$ \\
\hline 2 & 3.451 & $4.5 \%$ & 128 & 0.676 & $13.1 \%$ & $64+1$ & 0.600 & $11.6 \%$ \\
\hline 3 & 1.252 & $1.6 \%$ & 192 & 0.273 & $5.3 \%$ & $64-2$ & 0.201 & $3.9 \%$ \\
\hline 4 & 0.526 & $0.7 \%$ & & & & $64+2$ & 0.265 & $5.1 \%$ \\
\hline 5 & 0.273 & $0.4 \%$ & & & & & & \\
\hline
\end{tabular}

surfaces yields a very repeatable TE on a tooth to tooth basis, as highlighted by the tight 95\% confidence bounds in Fig. 19 . Repeatable oscillations on the high plateau of each TE curve is expected to be due to sub-micron waviness of tooth surfaces. A slight decrease of repeatability can be observed when a new tooth comes into contact; this is expected to be due to impulsive non-conjugate contact, happening because tooth deflections cause involute distortions. Such deviations from perfect involutes is however very limited and indeed TE converges towards zero for a very low torque value. This demonstrate very good confidence and accuracy margins provided by the combination of the ETM and the low-cost encoders. 


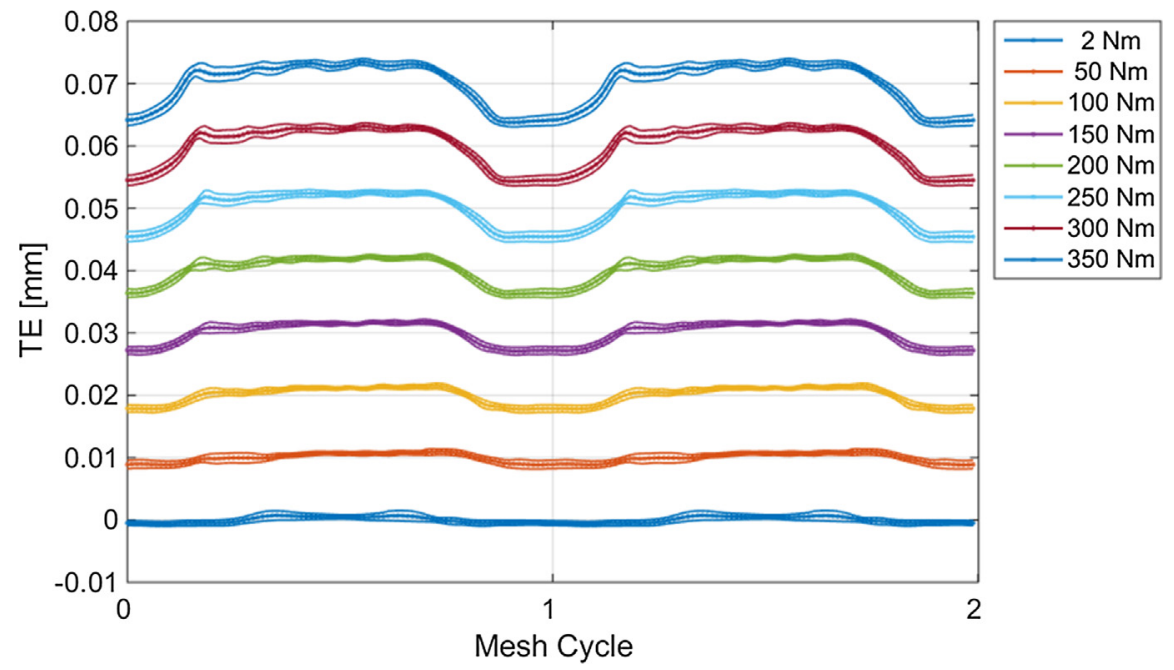

Fig. 19. Averaged TE on a tooth-to-tooth basis with $95 \%$ confidence bounds for two mesh cycles and varying torque.

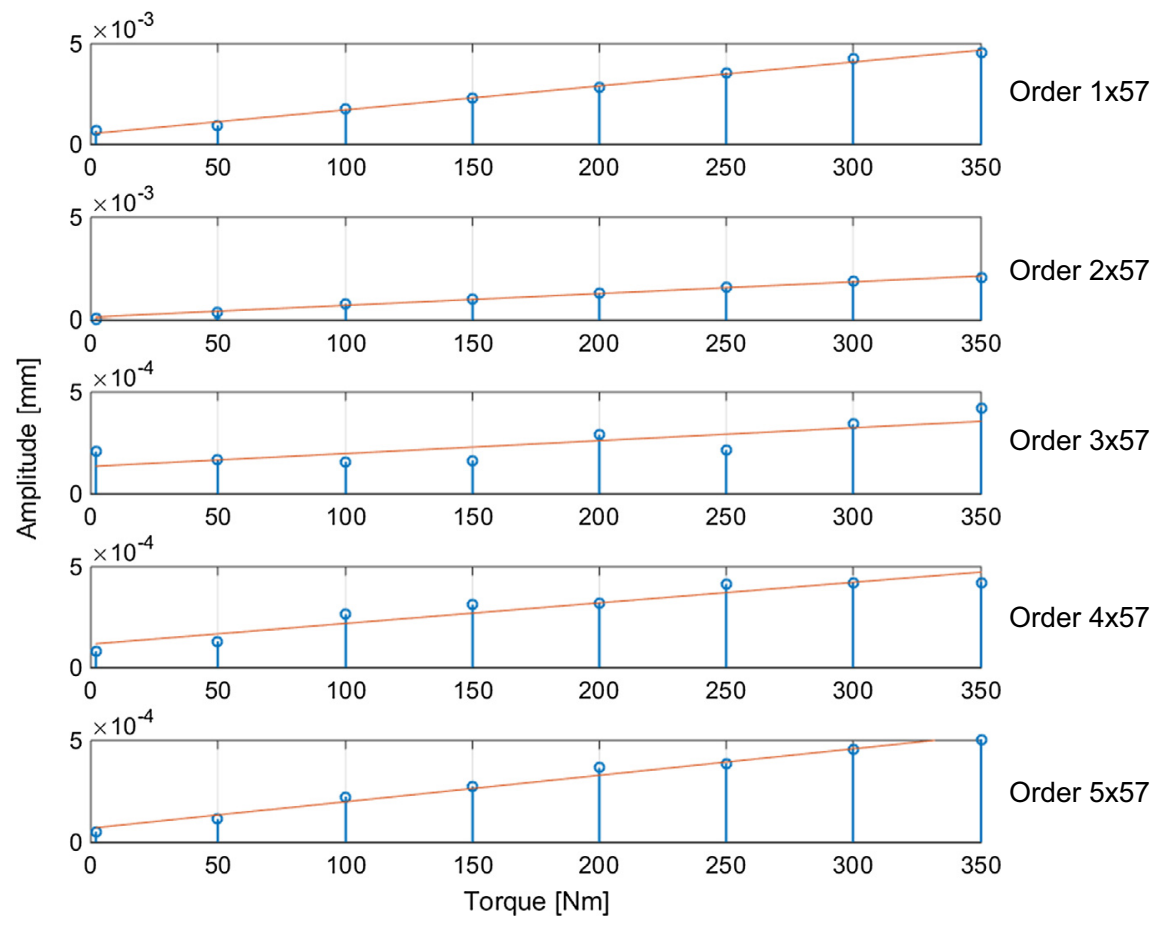

Fig. 20. Spectral amplitude for the first five meshing orders and best linear fit as a function of the applied torque (for orders 3,4 and 5 please notice the different y axis scale).

A better evaluation of measurement accuracy can be performed by looking at the order content of the TE as a function of torque (Fig. 20). Since no microgeometry modifications are applied, mesh deflections are expected to be substantially linear with load [54]. Linear trends are observed for all tooth orders with repeatable measurement of $0.05 \mu \mathrm{m}$.

After the consideration just outlined, the accuracy of the measurement procedure using the ETM can be considered validated for the precision gear pair test rig. The robustness of the procedure will be verified in the next section with an industrial application case. 


\section{Application to an all-electric vehicle gearbox}

\subsection{Gearbox and drive unit description}

The gearbox under investigation is a fixed gear ratio speed reducer used in the power unit of an all-electric vehicle. For confidentiality reasons internal or quantitative details of such power unit are not revealed. Such quantitative values are however not needed to discuss the installation and the main outcome of the measurement campaign. The speed reducer consists of a conventional three-shaft gearbox design two parallel helical gear pairs (Fig. 21).

\subsection{Test rig description and instrumentation}

Similar analyses as described in Section 3 have been performed for each shaft of the gearbox to select the encoder types and the number of angular divisions per encoder. Thanks to the advantages previously discussed, the ETM has been used together with low-cost digital encoders of type ERN 420. Signals are acquired and processed according to the measuring chain in Fig. 22.

A conventional mechanical power recirculation back to back test rig available at the OEM premises is used to measure the TE between each gear pair of the gearbox (Fig. 21), while the power unit is fully assembled. The decision to use a back to back test rig is mainly built on the smooth and exact torque loading and the advantages regarding encoder mounting. Two identical power units are used to create a torque loop between their input and output shafts. One power unit is referred to as the "slave" unit, since it is only needed to retain the load in the system. The power unit on the opposite end of the test rig is referred to as the "test" unit and is the one which is instrumented. The slave unit has its output shaft not only connected to the test unit but also to an electric motor which drives the system. The input shaft line is split and includes a mechanism to twist two shaft segments against each other in order to introduce load on the gears. A torque transducer is used to measure the imposed torque. To simplify mounting for the test unit a bellow coupling is used to connect its input shaft to the test rig, while the output shaft is connected via a cardan shaft. For reasons of alignment and for the reduction of torsional vibration interaction between the two power units an elastomer coupling is used to connect the slave unit.

\subsection{Measurement results}

Given the periodicity of tooth meshing vibrations with respect to shaft rotations, all data are analysed in angle domain rather than in time domain. Spectral analysis is typically performed by Order Tracking, with the exception of waterfall plots in case of speed run-up or run-down which usually provide a clearer identification of resonances when performed in frequency domain [65]. The discussion is limited for the scope of this article to the case of stationary conditions at a given speed. Measurements are first analysed in angle domain as directly obtained from angle differences. A typical result is shown in Fig. 23, where the long and short wave TE components can be clearly distinguished. One difference with results in Figs. 16 and 17 is that, given a non-integer gear ratio, the fundamental period of the long wave is given by the slowest shaft of the gear pair; for the same reason perfect periodicity is not achieved from cycle to cycle - theoretically the fundamental period would be the one to have the same two teeth mesh again. The same result is taken to order domain with FFT and, given the gearbox kinematics, shafts and gear orders are checked. This analysis allows tracing the excitation to each gear and each shaft in order to rate their relative contribution. Based on the order analysis, gear mesh orders can be isolated by filtering and analysed separately (Fig. 23). In this case, long and short wave component have been separated respectively below and above the third harmonic of the highest shaft order of the gear pair. In particular, tooth to tooth variability due to manufacturing and the effects of modulation can be evaluated. TE metrics can also be calculated to quantify the gear meshing

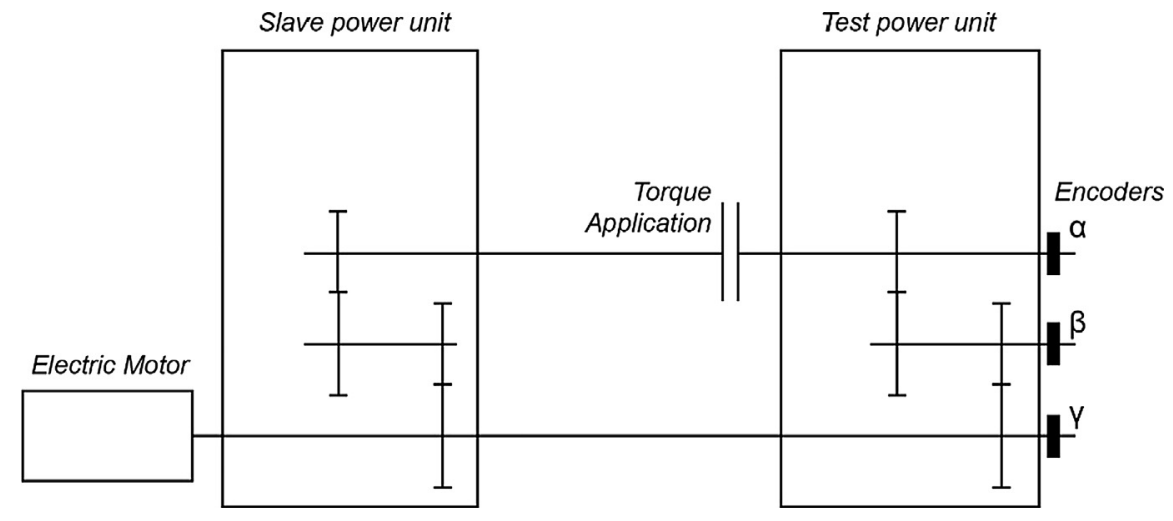

Fig. 21. Back to back test rig scheme. 


\begin{tabular}{|c|c|c|c|c|c|c|}
\hline Input Shaft Rotation & Angle $\alpha$ & $\begin{array}{l}\text { Optical Encoder } \\
\text { ERN } 420\end{array}$ & TTL Signal & \multirow{4}{*}{$\begin{array}{l}\text { Acquisiton Card RV4: } \\
\text { 1. Signal Conditioning } \\
\text { 2. Anti-Alias Filtering } \\
\text { 3. ADC Conditioning } \\
\text { 4. } 2 x \text { Upsampling } \\
\text { 5. } 32 \text { Point Lagrange Interpolation } \\
\text { 6. Linear Interpolation }\end{array}$} & \multirow{4}{*}{ Tacho Moments } & \multirow{4}{*}{$\begin{array}{l}\text { LMS Test. Lab: } \\
\text { 1. } \text { TE }_{\text {First Stage }}=\mathrm{i}_{1} \times \beta-\alpha \\
\text { 2. TE } \mathrm{E}_{\text {Second Stage }}=\mathrm{i}_{2} \times \gamma-\beta \\
\text { 3. Filtering } \\
\text { 4. FFT } \\
\text { 5. Graphical Representation }\end{array}$} \\
\hline Int. Shaft Rotation & Angle $\beta$ & Optical Encoder & TTL Signal & & & \\
\hline & & & & & & \\
\hline Out. Shaft Rotation & Angley & $\begin{array}{l}\text { Optical Encoder } \\
\text { ERN } 420\end{array}$ & TTL Signal & & & \\
\hline
\end{tabular}

Fig. 22. Measuring chain for signal acquisition and processing for the drive unit.
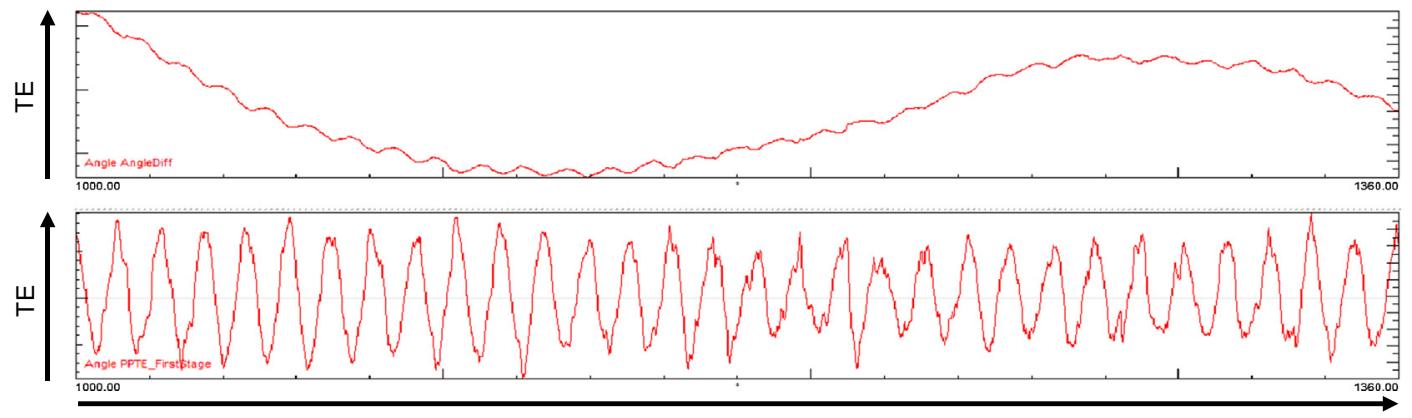

Fig. 23. Measured TE on the power unit. Upper: unprocessed TE, Lower: filtered short wave component.

excitation. Peak to peak TE value of the short wave component, defined as the difference between maximum and minimum TE value, is one of the most common TE metrics.

Peak to peak TE value shows to be repeatable within $0.04 \mu \mathrm{m}$ after 5 different measurement runs at given torque.

Angle synchronous averaging can then be applied after filtering the long wave TE components. The averaging period can be either one rotation of the driving shaft or one rotation of the driven shaft. The main effect of averaging will be a reduced tooth-to-tooth variability due to manufacturing errors which will simplify considerations on TE optimization due to microgeometry modifications. An example of averaged TE at different torques is provided in Fig. 24. The non-linear dependence of TE on torque, mainly due to profile microgeometry, can be observed both in the TE amplitude and in the shape of the waveform.

An example of the usefulness of the peak to peak as a metric to quantify TE variability is reported in Fig. 25. The non-linear dependence of TE peak to peak with respect to transmitted torque has a trend which correlates well with gearbox
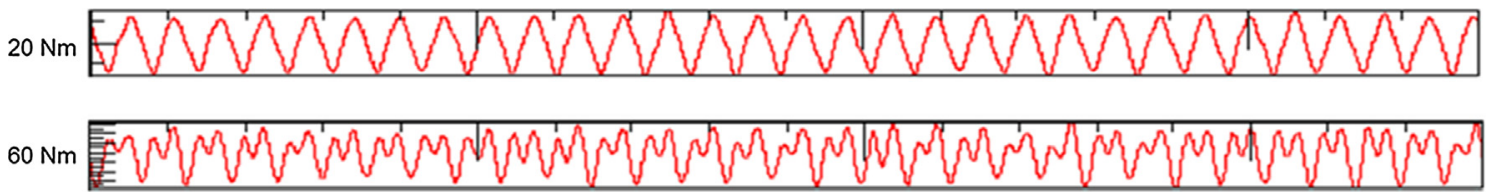

Fig. 24. Averaged TE measurements for different torque values.

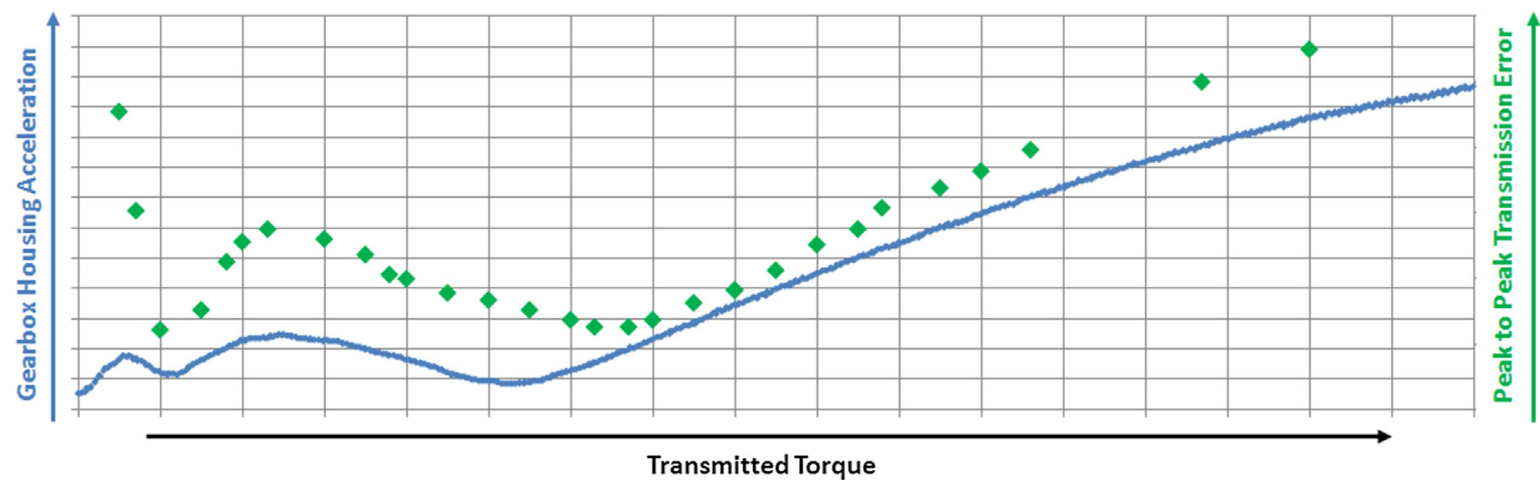

Fig. 25. Comparison between peak to peak TE value (dotted green) and gearbox housing acceleration RMS (blue) as a function of the transmitted torque. (For interpretation of the references to colour in this figure legend, the reader is referred to the web version of this article.) 
normal acceleration RMS value at key housing locations. Local minima happen to be in correspondence of torques where the profile modification is optimal.

Different profile microgeometry modifications lead to different optimal torques. The proposed measurement methodology is therefore well suited for evaluation of different design variants and for correlation towards simulations. Ultimately, TE proves to be a good quantitative indicator for gearbox $\mathrm{NVH}$.

\section{Conclusions}

Together with an overview of different gearbox NVH aspects, this article provides first a discussion and literature references to explain why gear Transmission Error analysis is useful to understand the causes and to tackle gear whine (tonal noise). Given the current limitations on gear modelling techniques at a system-level, emphasis is then posed on TE measurement. Two experimental test facilities were then used to draw conclusions on TE measurement: a precision gear pair test rig available at Siemens and an industrial test rig available at the premises of an OEM. The former was used to compare the traditional Direct Method (DM), through high-resolution analogue encoders, and the Elapsed Time Method (ETM), through lowcost digital encoders. The latter was used to measure TE on the power unit of an all-electric vehicle.

From a detailed analysis on the precision gear pair test rig, the ETM proves to yield accurate and repeatable TE measurement with a resolution in the order of a hundredth of a micron (Sections 3.1.1 and 4). Besides its accuracy, the ETM proves to have several advantages over the traditional DM:

- a speed range which is in the order of a hundred times or more (3.2.1);

- encoder instrumentation which is considerably less sensitive to misalignments and more suitable for industrial tolerances (3.2.2);

- reduced costs of instrumentation.

It is worth to note that the specifications of the high-performance general-purpose acquisition system used in this article allow reaching such accuracy and do not limit maximum speed. Speed limitations are in fact due to the encoders.

Experiments on the OEM test rig show robustness of the method for industrial application. Repeatability in a range which is similar to the precision gear pair test rig shows that industrial assembly tolerances do not degrade the quality of measurement. In fact, TE measurements prove valuable for quantitative performance comparison of gearbox design variants and in view of correlation towards system-level simulations. More in particular TE peak to peak value for the short wave component, selected as a metric of TE variability, proves to be a meaningful indicator for gearbox NVH: such metric and normal acceleration at key gearbox housing locations show a similar non-linear trend with respect to torque.

\section{Acknowledgements}

The results described in this article have been obtained through research activities funded by the People Programme (Marie Curie Actions) of the 7th Framework Programme of the European Union FP7/2007-2013, under the contract with the Research Executive Agency (REA) n. 324336 and the related research project DEMETRA - "Design of Mechanical Transmissions: Efficiency, Noise and Durability Optimization" (www.fp7demetra.eu).

\section{References}

[1] S. Theodossiades, O. Tangasawi, H. Rahnejat, Multi-physics Approach for Analysis of Transmission Rattle, Tribology and Dynamics of Engine and Powertrain: Fundamentals, Applications and Future Trends, Woodhead Publishing, 2010, pp. 878-913.

[2] J.-L. Dion, S. Le Moyne, G. Chevallier, H. Sebbah, Gear impacts and idle gear noise: experimental study and non-linear dynamic model, Mech. Syst. Sig. Process. 23 (2009) 2608-2628, https://doi.org/10.1016/j.ymssp.2009.05.007.

[3] M. Bozca, Torsional vibration model based optimization of gearbox geometric design parameters to reduce rattle noise in an automotive transmission, Mech. Mach. Theory 45 (2010) 1583-1598, https://doi.org/10.1016/j.mechmachtheory.2010.06.014.

[4] P. Charles, J.K. Sinha, F. Gu, L. Lidstone, A.D. Ball, Detecting the crankshaft torsional vibration of diesel engines for combustion related diagnosis, J. Sound Vib. 321 (2009) 1171-1185, https://doi.org/10.1016/j.jsv.2008.10.024.

[5] K. Janssens, L. Britte, Comparison of Torsional Vibration Measurement Techniques, Advances in Condition Monitoring of Machinery in Non-Stationary Operations, Springer, 2014, pp. 453-463.

[6] J.D. Smith, Gear Noise and Vibration, Marcel Dekker, 2003.

[7] S.L. Harris, Dynamic loads on the teeth of spur gears, Proc. IMechE 172 (1957) 87-112.

[8] R.W. Gregory, S.L. Harris, R.G. Munro, Dynamic behaviour of spur gears, Proc. IMechE 178 (1963) $207-226$.

[9] Y. Guo, T. Eritenel, T.M. Ericson, R.G. Parker, Vibro-acoustic propagation of gear dynamics in a gear-bearing-housing system, J. Sound Vib. 333 (2014) 5762-5785, https://doi.org/10.1016/j.jsv.2014.05.055.

[10] R.G. Parker, S.M. Vijayakar, T. Imajo, Non-linear dynamic response of a spur gear pair: modelling and experimental comparisons, J. Sound Vib. 237 (3) (2000) 435-455.

[11] International Standard ISO 6336, Calculation of load capacity of spur and helical gears.

[12] T.F. Conry, A. Seireg, A mathematical programming technique for the evaluation of load distribution and optimal modifications for gear systems, J. Eng. Ind. (1973) 1115-1122.

[13] H.N. Ozguven, D.R. Houser, Mathematical models used in gear dynamics - a review, J. Sound Vib. 121 (3) (1988) $383-411$.

[14] J. Wang, I. Howard, Finite element analysis of high contact ratio spur gears in mesh, J. Tribol. 127 (2005) 469-483.

[15] A. Cardona, Three-dimensional gears modelling in multibody systems analysis, Int. J. Numer. Meth. Eng. 40 (1997) $357-381$. 
[16] A. Palermo, D. Mundo, R. Hadjit, W. Desmet, Multibody element for spur and helical gear meshing based on detailed three-dimensional contact calculations, Mech. Mach. Theory 62 (2013) 13-30.

[17] A. Andersson, L. Vedmar, A dynamic model to determine vibrations in involute helical gears, J. Sound Vib. 260 (2003) $195-212$.

[18] S.M. Vijayakar, A combined surface integral and finite element solution for a three-dimensional contact problem, Int. J. Numer. Meth. Eng. 31 (1991) $524-546$.

[19] B. Blockmans, T. Tamarozzi, F. Naets, W. Desmet, A nonlinear parametric model reduction method for efficient gear contact simulations, Int. J. Numer. Meth. Eng. 102 (2015) 1162-1191.

[20] C. Brecher, C. Gorgels, J. Hesse, M. Hellmann, Dynamic transmission error measurements of a drive train, Prod. Eng. Res. Devel. 5 (2011) 321-327, https://doi.org/10.1007/s11740-011-0310-5.

[21] R. Munro, A review of the single flank method for testing gears, Ann. CIRP 28 (1979).

[22] V.K. Tamminana, A. Kahraman, S. Vijayakar, A study of the relationship between the dynamic factors and the dynamic transmission error of spur gear pairs, J. Mech. Des. 129 (2007) 75, https://doi.org/10.1115/1.2359470.

[23] C. Gosselin, T. Guertin, D. Remond, Y. Jean, Simulation and experimental measurement of the transmission error of real hypoid gears under load, J. Mech. Des. 122 (2000) 109-122.

[24] R.G. Munro, D. Palmer, L. Morrish, An experimental method to measure gear tooth stiffness throughout and beyond the path of contact, Proc. Instit. Mech. Eng., Part C: J. Mech. Eng. Sci. 215 (2001) 793-803.

[25] D.R. Houser, G.W. Blankenship, Methods for Measuring Gear Transmission Error Under Load and at Operating Speeds, SAE International, Warrendale, PA, 1989, http://papers.sae.org/891869/.

[26] R.G. Munro, A review of the theory and measurement of gear transmission error, IMechE, 1990.

[27] M.P. Pierz, Development of a precision variable load transmission error test device, SAE Technical Paper, 2001. http://papers.sae.org/2001-01-1491/.

[28] J.H. Yoon, B.J. Choi, I.H. Yang, J.E. Oh, Deflection test and transmission error measurement to identify hypoid gear whine noise, Int.J Automot. Technol. 12 (2011) pp. 59-66. doi: 10.1007/s12239-011-0008-X.

[29] R.J. White, Exploration of a Strategy for Reducing Gear Noise in Planetary Transmissions and Evaluation of Laser Vibrometry as a Means for Measuring Transmission Error, Case Western Reserve University, 2006.

[30] R. White, V. Palan, Measurement of transmission error using rotational laser vibrometers, in: ASME 2007 International Design Engineering Technical Conferences and Computers and Information in Engineering Conference, American Society of Mechanical Engineers, 2007 , pp. 527-545.

[31] Dr. Johannes Heidenhain GmbH, Angle encoders catalog, 2015.

[32] J.D. Smith, Gear transmission error accuracy with small rotary encoders, Proc. Instit. Mech. Eng., Part C: J. Mech. Eng. Sci. 201 (1987) 133-135, https:// doi.org/10.1243/PIME_PROC_1987_201_096_02.

[33] J.D. Smith, Practical rotary encoder accuracy limits for transmission error measurement, Proc. Instit. Mech. Eng., Part C: J. Mech. Eng. Sci. 205 (1991) 431-436, https://doi.org/10.1243/PIME_PROC_1991_205_141_02.

[34] S. Kurokawa, Y. Ariura, Y. Matsukawa, T. Doi, Evaluation of gear engagement accuracy by Transmission Error with sub-microradian resolution, Int. J. Surf. Sci. Eng. 3 (2009) 160-177.

[35] D. Remond, J. Mahfoudh, From transmission error measurements to angular sampling in rotating machines with discrete geometry, Shock Vib. 12 (2005) 149-161, https://doi.org/10.1155/2005/205291.

[36] D. Remond, Practical performances of high-speed measurement of gear transmission error or torsional vibrations with optical encoders, Meas. Sci. Technol. 9 (1998) 347.

[37] J.D. Smith, Accuracy of small encoders for gear transmission errors, Proc. Instit. Mech. Eng., Part C: J. Mech. Eng. Sci. 215 (2001) $995-998$.

[38] S. Du, R.B. Randall, Encoder error analysis in gear transmission error measurement, Proc. Instit. Mech. Eng., Part C: J. Mech. Eng. Sci. 212 (1998) 277285, https://doi.org/10.1243/0954406981521213.

[39] P.J. Sweeney, R.B. Randall, Gear transmission error measurement using phase demodulation, Proc. Instit. Mech. Eng., Part C: J. Mech. Eng. Sci. 210 (1996) 201-213, https://doi.org/10.1243/PIME_PROC_1996_210_190_02.

[40] S. Seidlitz, R.J. Kuether, M.S. Allen, Comparison of noise floors of various torsional vibration sensors, in: Topics in Modal Analysis II, Volume 6, Springer, 2012, pp. 153-168. http://link.springer.com/chapter/10.1007/978-1-4614-2419-2_14.

[41] M.R. Kang, A. Kahraman, Measurement of vibratory motions of gears supported by compliant shafts, Mech. Syst. Sig. Process. 29 (2012) 391-403, https://doi.org/10.1016/j.ymssp.2011.11.007.

[42] Y. Li, F. Gu, G. Harris, A. Ball, N. Bennett, K. Travis, The measurement of instantaneous angular speed, Mech. Syst. Sig. Process. 19 (2005) 786-805, https://doi.org/10.1016/j.ymssp.2004.04.003.

[43] S. Sasaoka, Measurement Technique for Loaded Gear Transmission Error, SAE Technical Paper, 1997. http://papers.sae.org/970973/.

[44] Antonio's PhD thesis.

[45] S.P. Radzevich, Dudley's Handbook of Practical Gear Design and Manufacture, CRC Press, 2012.

[46] G.W. Blankenship, A. Kahraman, Steady state forced response of a mechanical oscillator with combined parametric excitation and clearance type nonlinearity, J. Sound Vib. 185 (1995) 743-765.

[47] R.G. Parker, S.M. Vijayakar, T. Imajo, Non-linear dynamic response of a spur gear pair: modelling and experimental comparisons, J. Sound Vib. 237 (2000) 435-455, https://doi.org/10.1006/jsvi.2000.3067.

[48] S. Theodossiades, S. Natsiavas, Non-linear dynamics of gear-pair systems with periodic stiffness and backlash, J. Sound Vib. 229 (2000) 287-310, https://doi.org/10.1006/jsvi.1999.2490.

[49] A. Kahraman, On the response of a preloaded mechanical oscillator with a clearance: period-doubling and chaos, Nonlinear Dyn. 3 (1992) 183-198.

[50] J. Lin, R.G. Parker, Mesh stiffness variation instabilities in two-stage gear systems, J. Vib. Acoust. 124 (2002) 68, https://doi.org/10.1115/1.1424889.

[51] A. Toso, F. Van Wermeskerken, N. Cappellini, G. Heirman, On the effect of lightweight gear blank topology on transmission dynamics, in: Proceedings of the ASME 2015 International Design Engineering Technical Conferences \& Computers and Information in Engineering Conference IDETC/CIE 2015, August 2-5, 2015, Boston, USA.

[52] ISO Standard, ISO 21771:2007, Gears - Cylindrical involute gears and gear pairs - Concepts and geometry.

[53] J.W. Vriesen, Topographical tooth corrections for noise reduction without load capacity loss, in: Proceedings of the VDI International Gear Conference 2013, October 7-9, Munich, Germany.

[54] A. Kahraman, G.W. Blankenship, Effect of involute tip relief on dynamic response of spur gear pairs, J. Mech. Des. 121 (1999) 313-315, https://doi.org/ $10.1115 / 1.2829460$

[55] M. Hotait, A. Kahraman, Experiments on root stresses of helical gears with lead crown and misalignments, J. Mech. Des. 130 (2008) 074502, https://doi. org/10.1115/1.2931127.

[56] M. Inalpolat, M. Handschuh, A. Kahraman, Influence of indexing errors on dynamic response of spur gear pairs, Mech. Syst. Sig. Process. 60 (2015) 391405.

[57] ISO Standard, ISO 1328-1:2013, Cylindrical gears - ISO system of flank tolerance classification.

[58] A. Palermo, J. Anthonis, D. Mundo, W. Desmet, A novel gear test rig with adjustable shaft compliance and misalignments part I: design, in: Advances in Condition Monitoring of Machinery in Non-Stationary Operations, Springer, 2014, pp. 497-506.

[59] A. Palermo, J. Anthonis, D. Mundo, W. Desmet, A novel gear test rig with adjustable shaft compliance and misalignments. Part II: instrumentation, in: Advances in Condition Monitoring of Machinery in Non-Stationary Operations, Springer, 2014, pp. 507-515.

[60] JCGM 200:2012, International Vocabulary of Metrology - Basic and general concepts and associated terms, 3rd edition, http://www.bipm.org/en/ publications/guides/vim.html.

[61] Dr. Johannes Heidenhain GmbH, Rotary encoders catalog, 2015. 
[62] Siemens PLM, LMS SCADAS product brochure, 2015.

[63] Siemens PLM, LMS SCADAS RV4 module specification datasheet, 2015.

[64] ISO Standard, ISO/TR 10064, Cylindrical gears - Code of inspection practice.

[65] Q. Leclère, L. Pruvost, E. Parizet, Angular and temporal determinism of rotating machine signals: the diesel engine case, Mech. Syst. Sig. Process. 24 (2010) 2012-2020, https://doi.org/10.1016/j.ymssp.2010.05.006. 